\title{
Semantics for Intuitionistic Arithmetic based on Tarski Games with retractable moves
}

\author{
Stefano Berardi \\ http://www.di.unito.it/ stefano \\ C.S. Dept., University of Torino, Italy
}

\begin{abstract}
We define an effective, sound and complete game semantics for $\mathrm{HA}_{\mathrm{inf}}$, Intuitionistic Arithmetic with $\omega$-rule. Our semantics is equivalent to the original semantics proposed by Lorentzen [6], but it is based on the more recent notions of "backtracking" ([5], [2]) and of isomorphism between proofs and strategies ([8]). We prove that winning strategies in our game semantics are tree-isomorphic to the set of proofs of some variant of $\mathrm{HA}_{\mathrm{inf}}$, and that they are a sound and complete interpretation of $\mathrm{HA}_{\text {inf }}$.
\end{abstract}

\section{Why game semantics of Intuitionistic Arithmetic?}

In [7], S.Hayashi proposed the use of an effective game semantics in his Proof Animation project. The goal of the project is "animating" (turning into algorithms) proofs of program specifications, in order to find bugs in the way a specification is formalized. Proofs are formalized in classical Arithmetic, and the method chosen for "animating" proofs is a simplified version of Coquand's game interpretation ([4], [5]) of $\mathrm{PA}_{\mathrm{inf}}$, classical arithmetic with $\omega$-rule. The interest of the game interpretation is that it interprets rules of classical arithmetic by very simple operations, like arithmetical operation, reference to a pointer, adding and removing elements to a stack.

Coquand, however, defined implication $A \rightarrow B$ as classical implication, as " $A$ is false or $B$ is true". In real proofs, instead, we often use the constructive definition of implication $A \Rightarrow B$, which is: "assume $A$ in order to prove $B$ ". $A \Rightarrow B$ is classically equivalent to " $A$ is false or $B$ is true", but this means that in order to interpret a proof in Coquand's semantics we have first to modify it. If we want some control and understanding of the algorithm we extract from a proof, instead, it is crucial to animate the original proof.

In this paper we adapt Coquand's game semantics of $\mathrm{PA}_{\mathrm{inf}}$ to game semantics of Intuitionistic Arithmetic $\mathrm{HA}_{\mathrm{inf}}$ with intuitionistic implication $\Rightarrow$. Our semantics is equivalent to the original Lorentzen's game semantics [6], and also bears some similarity with Hyland-Ong game semantics for simply typed lambda terms [10]. The main difference between our semantics and Lorentzen's semantics is that we do no not add dummy moves when interpreting connectives, but when interpreting implication 
(see $\S 4.4$ for a discussion). Reducing the number of dummy moves is crucial in order to make evident the relationship between a game strategy and the intuitionistic proof interpreted by the strategy. The main difference between our semantics and Hyland-Ong's semantics is, instead, that we consider all connectives as Lorentzen did. In this way the difference between the game interpretation for implication and the game interpretation for all other connectives becomes evident.

The game interpretation of the intuitionistic implication introduced in this paper aims to be one step in the Proof Animation project. What is still missing are game semantics combining all features of real proofs: classical logic, intuitionistic implication, cut rule, induction rule, and so forth. We claim that the semantics introduced in this paper can also be used to interpret Cut rule through the notion of dialogue, as it was done by Coquand, Hyland-Ong and Herbelin. We did not include Cut rule for reason of space. Even without Cut rule, we can use our semantic to interpret the evidence provided by an intuitionistic proof in term of very simple operations, without blurring the relation with the proof structure during the interpretation.

\subsection{The plan of the paper}

This is the plan of the paper. In $\S 2$ we introduce the language of arithmetic. In $\S 3$ we introduce our game semantics. In $\S 4, \S 5$ we discuss our definition of game, comparing it with Lorentzen's. In $\S 6$ we introduce $\mathrm{HA}_{\text {inf }}$, intuitionistic arithmetic with $\omega$-rule. In $\S 7,8$ we prove that winning strategies of our semantics are tree-isomorphic with the proofs of some variant of $\mathrm{HA}_{\mathrm{inf}}$, and that our game semantics is sound and complete for $\mathrm{HA}_{\mathrm{inf}}$. In $\S 9$ we introduce one example of winning strategy in our game semantics. This winning strategy can also be seen as a proof in $\mathrm{HA}_{\mathrm{inf}}$. In $\S 10$ we introduce one example of play which uses the winning strategy defined in $\S 9$.

\section{The language of Arithmetic}

In this section we introduce a language $\mathrm{L}_{\mathrm{HA}}$ for first order arithmetic, the notion of judgement, and the notion of sequent. In the next section we define our game semantics.

L HA has a connective $A \Rightarrow B$ denoting intuitionistic implication. We also introduce a "game language" $\mathrm{L}_{\mathrm{G}} \supset \mathrm{L}_{\mathrm{HA}}$ for game semantics. The formulas of $\mathrm{L}_{G}$ denote games interpreting formulas of $\mathrm{L}_{\mathrm{HA}}$. Each connective of $\mathrm{L}_{\mathrm{HA}}$ corresponds to some operator defining games in $\mathrm{L}_{\mathrm{G}}$, an operator which we will denote with the same symbol. In this way each formula of $\mathrm{L}_{\mathrm{HA}}$ will be also considered as a denotation for some game in $\mathrm{L}_{\mathrm{HA}}$ interpreting it. The only difference between $\mathrm{L}_{\mathrm{G}}$ and $\mathrm{L}_{\mathrm{HA}}$ is that $\mathrm{HA}$ has one extra connective $\rightarrow$. The connective $\rightarrow$ denotes one binary operator on games, used as an intermediate step in the interpretation of intuitionistic implication $\Rightarrow$ (see $\S 4.4$ for a discussion).

We divide the formulas of the game language $\mathrm{L}_{\mathrm{G}}$ into disjunctive and conjunctive, by generalizing the usual distinction between disjunctive 
and conjunctive formulas we have in Logic. We consider $A \Rightarrow B$ a conjunction, and $A \rightarrow B$ a disjunction. The language $\mathrm{L}_{\mathrm{HA}}$, for intuitionistic arithmetic, consists of all formulas of $\mathrm{L}_{\mathrm{G}}$ which are $\rightarrow$-free.

Definition 1. $-\mathrm{L}_{\mathrm{G}}$ is the first order language including a r.e. set of function symbols for recursive functions (at least $0, S,+, *$ ), and a r.e. set of predicate symbols for recursive predicates (at least $<,=$ ), and the connectives $T, F, \wedge, \vee, \neg, \Rightarrow, \forall, \exists$ and $\rightarrow$. We call $T, F$ "true" and "false", and we consider them different from all atomic formulas.

- $\mathrm{L}_{\mathrm{HA}}$, the language of intuitionistic arithmetic, is the sub-language of $\mathrm{L}_{\mathrm{G}}$ consisting of all formulas which are $\rightarrow$-free. $\mathrm{L}^{+}$, the language of positive formulas, is the sub-language of $\mathrm{L}_{\mathrm{HA}}$ consisting of all formulas which are $\neg, \Rightarrow, \rightarrow$-free. $\mathrm{L}_{\mathrm{G} 0}, \mathrm{~L}_{\mathrm{HAO}}, \mathrm{L}_{0}^{+}$are the sets of closed formulas of $\mathrm{L}_{\mathrm{G}}, \mathrm{L}_{\mathrm{HA}}, \mathrm{L}^{+}$.

- If $A \in \mathrm{L}_{\mathrm{G}}$ is $F$, or is atomic, or starts with $\vee, \rightarrow, \exists$, we say that $A$ is disjunctive. If $A \in \mathrm{L}_{\mathrm{G} 0}$ is $T$, or starts with $\wedge, \Rightarrow, \neg, \forall$, we say that $A$ is conjunctive.

We consider $T$ conjunctive because the constant true is equivalent to an empty conjunction. We consider $F$ disjunctive because the constant false is equivalent to an empty disjunction. We consider (arbitrarily) all atomic formulas to be disjunctive. The only new and non-trivial choice is considering $A \Rightarrow B$ conjunctive and $A \rightarrow B$ disjunctive: we discuss this choice in $\S 4.4$. $\neg A$ is taken conjunctive by analogy with $A \Rightarrow F$, which is equivalent to $\neg A$. We use $t, u, v, \ldots$ to denote closed terms of $\mathrm{L}_{\mathrm{G} 0}$. We use $A, B, C, D, A_{1}, B_{1}, C_{1}, D_{1}, \ldots$ to denote closed formulas of $\mathrm{L}_{\mathrm{G} 0}$, and $a, b, c, \ldots$ for atomic closed formulas.

We consider the usual subformula relation between closed formulas, with the additional clauses: the only immediate subformula of $A \Rightarrow B$ is $A \rightarrow$ $B$, and: the only subformula of $a$ atomic is $T$ or $F$, according if $a$ is true or false.

Definition 2. (Immediate Subformula relation $<_{1}$ over $\mathrm{L}_{\mathrm{G} 0}$ )

- $T, F$ have no immediate subformula. If $A \in \mathrm{L}_{\mathrm{GO}}$ is atomic, the only immediate subformula of $A$ is $T$ if $A$ is true, and $F$ if $A$ is false. The immediate subformulas of $A \vee B, A \wedge B, A \rightarrow B$ are $A, B$. The only immediate subformula of $A \Rightarrow B$ is $A \rightarrow B$. The only immediate subformula of $\neg A$ is $A$. The immediate subformulas of $\forall x . A[x], \exists x . A[x]$ are all $A[t]$ for $t$ closed term of $\mathrm{L}_{\mathrm{G}}$. If $A$ is an immediate subformula of $B$, we write $A<_{1} B$ (or $B>_{1} A$ ). The subformula relation $<$ is the transitive closure of $<_{1}$.

- Assume $A<_{1} C$. If $C=\neg A, A \rightarrow B$, we say that $A$ is negative in $C$. In all other cases, we say that $A$ is positive in $C$.

- An occurrence of a subformula $B$ in $A$ is any sequence $A_{0}, \ldots, A_{n}$ with $A_{0}=A$, and $A_{i}>_{1} A_{i+1}$ for all $i<n$, and $A_{n}=B$. The occurrence is positive if $A_{i+1}$ is negative in $A_{i}$ for an even number of $i$, the occurrence is negative, if $A_{i+1}$ is negative in $A_{i}$ for an odd number of $i$.

We use the informal notion of tree and all tree terminology (children, father, ascendant, descendant, branch, leaf). The subformula tree of 
$C \in \mathrm{L}_{\mathrm{GO}}$ is the tree of all subformula occurrences of $C$, ordered by the subformula relation <. If $C \in \mathrm{L}_{\mathrm{HA}}$, then $C$ by definition is $\rightarrow$-free. Yet, the subformula tree of $C$ in $\mathrm{L}_{\mathrm{GO}}$ can include occurrences of some $A \rightarrow B$, the children of the occurrences of some $A \Rightarrow B$ in the tree. The only difference between an occurrence of $A \Rightarrow B$ and an occurrence of $A \rightarrow B$ is that the former is conjunctive while the latter is disjunctive. This duplication of nodes looks useless, but it will play a crucial role in $\S 4.4$. If we restrict the subformula relation to $\mathrm{L}_{\mathrm{HA}}$, then we skip $A \rightarrow B$. In this case the immediate subformulas of $A \Rightarrow B$ are $A, B$, as expected. We now formally define the notion of judgement and sign of a judgement.

Definition 3. (Judgement and Sign of a Judgement) Let $A \in \mathrm{L}_{\mathrm{G} 0}$.

1. We call t. A and f.A judgements, and we read them " $A$ is true", "A is false". We call s the sign of $s A$, and we say the sign is positive if $s=\mathrm{t}$., is negative if $s=\mathrm{f}$. . We denote by $s^{\perp}$ the opposite sign of $s: s^{\perp}=\mathrm{f}$. if $s=\mathrm{t}$., and $s^{\perp}=\mathrm{t}$. if $s=\mathrm{f}$..

2. We say that t.A is a disjunctive judgement (respectively, a conjunctive judgement) if $A$ is a disjunctive formula (respectively, a conjunctive formula).

3. We say that $\mathrm{f}$. A is a disjunctive judgement (respectively, a conjunctive judgement) in the opposite case, namely, if $A$ is a conjunctive formula (respectively, a disjunctive formula).

By switching the sign of a formula we switch conjunctive and disjunctive judgements. For instance, t. $A \Rightarrow B$ is conjunctive, while $\mathrm{f} . A \Rightarrow B$ is disjunctive. There is a sub-judgement relation analogous to the sub-formula relation, except that the judgements have opposite signs whenever the subformula is negative.

Definition 4. (Immediate sub-judgement relation) Let $A \in \mathrm{L}_{\mathrm{G} 0}$. We say that $s^{\prime} A^{\prime}$ is an immediate sub-judgement of $s A$, and we write $s^{\prime} A^{\prime}<_{1} s A$, if $A^{\prime}<_{1} A$, and: $s^{\prime}=s$ if $A^{\prime}$ is positive in $A$, and $s^{\prime}=s^{\perp}$ if $A^{\prime}$ is negative in $A$. The sub-judgement relation $<$ is the transitive closure of $<_{1}$.

For instance, the immediate sub-judgements of t. $A \rightarrow B$ are $\mathrm{f} . A$ and t. $B$, while the immediate sub-judgements of $\mathrm{f} . A \rightarrow B$ are t. $A$ and f. $B$. If we restrict the sub-judgement relation to judgements of $\mathrm{L}_{\mathrm{HA}}$, then the immediate sub-judgements of t. $A \Rightarrow B$ are f. $A$, t. $B$, as expected.

We will now introduce a notion of pointed sequent: sequents having one "active formula" in evidence. We first recall what (finite) multisets are. A multiset is a set with possibly repetitions, in which elements $x_{1}, \ldots, x_{n}$ are distinguished through the use of labels. We use positive integers as labels. We formalize a multiset $X$ with the set of its indexes paired with the corresponding elements: $X=\left\{\left\langle i_{1}, x_{1}\right\rangle, \ldots,\left\langle i_{n}, x_{n}\right\rangle\right\}$, with $0<i_{1}<$ $\ldots<i_{n}$. We often write $X=x_{1}, \ldots, x_{n}$, leaving $i_{1}, \ldots, i_{n}$ implicit: the actual indexing is irrelevant. We use $\Gamma, \Delta, \Gamma^{\prime}, \Delta^{\prime}, \ldots$ to denote multisets of closed formulas in $\mathrm{L}_{\mathrm{G} 0} .\{A\}$ is the multiset consisting of one pair $\langle j, A\rangle$ for some $j>0$. We denote the disjoint union of two multisets $\Gamma$ and $\Delta$ with $\Gamma, \Delta$. By renaming indexing, we can always assume two multisets are disjoint. We denote $\Gamma,\{A\}$ with $\Gamma, A$. 
Definition 5. (Intuitionistic Sequents and occurrences) Let $\Gamma=\left\{\left\langle j_{1}, A_{1}\right\rangle, \ldots,\left\langle j_{n}, A_{n}\right\rangle\right\}$ and $\{\langle j, D\rangle\}$ be two multisets over $\mathrm{L}_{\mathrm{G} 0}$, with disjoint set of indexes, and $0<j_{1}<\ldots<j_{n}$.

- An intuitionistic sequent on $\mathrm{L}_{\mathrm{G} O}$ is any pair $\Gamma \vdash\{D\}$. A sequent is on $\mathrm{L}_{\mathrm{HAO}}$ if all its formulas are.

- The indexing of $\Gamma \vdash\{D\}$ is the union of indexing of $\Gamma$ and $\{D\}$. We call the index $i>0$ of $A$ in $\Gamma \vdash D$ an occurrence of $A$ in $\Gamma \vdash D$.

- A pointed sequent is a pair $\langle\Gamma \vdash D, i\rangle$ of an intuitionistic sequent on $\mathrm{L}_{\mathrm{G} 0}$, and an occurrence $i>0$ of some $A$ in $\Gamma \vdash D$. We call $i$ the active occurrence of the sequent.

$-\langle\Gamma \vdash D, i\rangle$ has canonical indexing if $j=1, j_{1}=2, \ldots, j_{n}=n+1$. The canonical version $\left\langle\Gamma^{\prime} \vdash D^{\prime}, i^{\prime}\right\rangle$ of $\Gamma \vdash D$ is obtained by replacing $j$ with $1, j_{1}$ with $2, \ldots, j_{n}$ with $n+1$, and defining $i^{\prime}=1$ if $i=j$, and $i^{\prime}=k+1$ if $i=j_{k}$ for some $k$.

From now on, we usually consider intuitionistic pointed sequents, and we call them just "sequents", for short. The canonical indexing $\left\langle\Gamma^{\prime} \vdash D^{\prime}, i^{\prime}\right\rangle$ of $\langle\Gamma \vdash D, i\rangle$ is obtained by renaming the indexes of $\langle\Gamma \vdash D, i\rangle$. The two sequents will be equivalent in our semantics. Also any two sequents $\langle\Gamma \vdash D, i\rangle$ and $\langle\Gamma \vdash D, j\rangle$, having different active formulas, will be equivalent. In logic, the indexing of $\Gamma$, and the active formula of a sequent are an irrelevant information. We can drop them and we can write a sequent just as $\Gamma \vdash D$. However, we need the indexing of $\Gamma$ and the active formula when defining the game interpretation.

Occurrences of a formula in a sequent have a sign, according to the side of the sequent they belong to. The sign of an occurrence, like the sign of a judgement, switches conjunctive and disjunctive formulas.

Definition 6. (Sign of a formula in a sequent)

- A formula occurrence in $\Gamma \vdash D$ has sign $\mathrm{t}$. (is positive) if it is in the right-hand-side. It has sign $\mathrm{f}$. (is negative) if it is in the left-handside.

- Assume $A$ is a positive occurrence. Then $A$ is a disjunctive occurrence if $A$ is a disjunctive formula, and a conjunctive occurrence if $A$ is a conjunctive formula.

- Assume $A$ is a negative occurrence. Then $A$ is a disjunctive occurrence if $A$ is a conjunctive formula, and a conjunctive occurrence if $A$ is a disjunctive formula.

We introduce two operations on sequents. The operation $\langle\Gamma \vdash D, i\rangle+s A$ adds an active formula $A$ of $\operatorname{sign} s$ to the sequent $\langle\Gamma \vdash D, i\rangle$ (and removes $D$, if we add $A$ to the right-hand-side), and chooses some index $n+1$ for $A$. The operation $\langle\Gamma \vdash D, i\rangle+\operatorname{bck}(j)$ changes the active formula of $\langle\Gamma \vdash D, i\rangle$ from $i$ to $j$ (provided $j$ is an index of $\Gamma \vdash D$ ).

Definition 7. 1. For all positive integers $j$ we introduce the notation $\operatorname{bck}(j)$.

2. Moves is a set of operators on sequents, consisting of all judgements $s A$ and all notations bck $(j)$.

3. Assume $\langle\Gamma \vdash D, i\rangle$ is any sequent, having maximum index $n$. Let $A \in \mathrm{L}_{\mathrm{GO}}, j>0$. We define a sequent $\langle\Gamma \vdash D, i\rangle+m$ for any operator $m \in$ Moves, by cases over $m$, as follows. 
$-\langle\Gamma \vdash D, i\rangle+\mathrm{f} . A=\langle\Gamma, A \vdash D, n+1\rangle$ (the sequent has active formula $A$, of sign $\mathrm{f}$. , and index $n+1)$.

- $\langle\Gamma \vdash D, i\rangle+\mathrm{t} . A=\langle\Gamma \vdash A, n+1\rangle$ (the sequent has active formula $A$, of sign t., and index $n+1)$.

- $\langle\Gamma \vdash D, i\rangle+\operatorname{bck}(j)=\langle\Gamma \vdash D, j\rangle$ if $j$ is an index of $\Gamma \vdash D$, and $=\langle\Gamma \vdash D, n\rangle$ o.w.

The sequent $\langle\Gamma \vdash D, i\rangle+s A$ has the same indexing of $\langle\Gamma \vdash D, i\rangle$, plus the index $n+1$ for $A$, and minus the index of $D$ if $s=\mathrm{t}$.. The sequent $\langle\Gamma \vdash D, i\rangle+\operatorname{bck}(j)$ has the same indexing of $\langle\Gamma \vdash D, i\rangle$ 4. $\langle\Gamma \vdash D, i\rangle+\left\langle m_{1}, \ldots, m_{k}\right\rangle=\left(\ldots\left(\langle\Gamma \vdash D, i\rangle+m_{1}\right)+\ldots\right)+m_{k}$.

We denote the active formula of a sequent boldface, for instance: $\Gamma, \mathbf{A} \vdash$ $D$ for a negative occurrence of $A$, and $\Gamma, A \vdash \mathbf{D}$ for a positive occurrence of $D$. Any two sequents over $\mathrm{L}_{\mathrm{HA}}$, differing only for the active occurrence, like $\Gamma, \mathbf{A} \vdash D$ and $\Gamma, A \vdash \mathbf{D}$, will be equivalent in our game semantics (by Theorem 3). This is not always the case for a sequent of $\mathrm{L}_{\mathrm{G} 0}$.

\section{Game semantics for Arithmetical Formulas}

In this section we define our game semantics for formulas of $\mathrm{L}_{\mathrm{G} 0}$. In the next section we unfold our definition and discuss its consequences.

We interpret (pointed) sequents $\langle\Gamma \vdash D, i\rangle$ as games are between two sides, $\mathcal{E}$ and $\mathcal{A}$. $\mathcal{E}$ is a single, finite and fallible being we call Eloise, able to learn from her mistakes. $\mathcal{A}$ is a potentially infinite array of omniscient, infallible beings we call the Abelard's, one for each move of the play. The omniscience of the Abelard's compensates the ability of Eloise to learn from her mistakes. Having one Abelard for each move of the game, instead, is just a colorful way of saying that Abelard decides his next move by considering only the previous move of the play. Therefore the replies of Abelard to two different moves are independent each other, and we can imagine they are made by two different individuals. Eloise can use all previous moves of the play to decide her next move, therefore her moves are related each other and we image them as made by a single individual.

The play between $\mathcal{E}, \mathcal{A}$ is interpreted as a debate. The play between $\mathcal{E}$ and each Abelard is called a "thread" in the play (we use the word "thread" with its informal meaning in Computer Science). In each position of the play, $\mathcal{E}$ defends a thesis, and one Abelard attacks it, or vice versa. Moves done in defence of a thesis cannot be retracted, for all players. The weak player, $\mathcal{E}$, can retract finitely many times a move done while attacking a thesis of $\mathcal{A}$. The strong players, the Abelard's, can never retract a move (neither in attack, nor in defence). There is also a thesis for the whole play. $\mathcal{E}$ claims that some $\Gamma \vdash D$ is true, while the array of Abelard's claims that $\Gamma \vdash D$ is false. We interpret truth of $\Gamma \vdash D$ by the existence of a recursive winning strategy for $\mathcal{E}$ on the game associated to the pointed sequent $\langle\Gamma \vdash D, i\rangle$, for some $i$.

We first introduce plays and correct moves. Moves include moves of Tarski plays [9]. This kind of move is called a logical move, and denoted by a judgement $s A$. There is a new kind of moves, "backtracking", 
when $\mathcal{E}$ comes back to the move number $i$ of the play, retracts the move she did after it, and selects a new move, with the goal of learning better and better moves in this way. This kind of move is called a structural move, and denoted by bck $(i)$. The idea of backtracking is taken from [4], [5], [2] (a similar idea can be found in [10], where it is used to interpret $\lambda$-terms). However, backtracking in our game semantics for intuitionism has a severe limitation: $\mathcal{E}$ can backtrack to any judgement having a negative sign, but only to the last judgement having a positive sign.

Definition 8. (Moves, positions and plays)

1. The set of all moves is Moves (Def. 7.2). A logical move is any judgment $s$ A. A structural move is any notation $\mathrm{bck}(i)$.

2. drop $\notin$ Moves is a special symbol, meaning: "I quit".

3. A position $Q$ is any non-empty finite sequence over Moves, starting with some t.A. We write $P \leq Q$ if $P$ is a prefix of $Q$.

4. A finished play $P$, or just a "play" for short, is either a sequence $Q$, drop, for some position $Q$, or an infinite sequence over Moves, starting with some t.A.

5. If $Q$ is any position, the indexing of logical moves (or judgements) in $Q$ is: 1 for the first logical move, 2 for the second one, and so forth.

Informally, we could describe a position as an "unfinished play". We define now the player $\mathcal{E} / \mathcal{A}$ moving from a given position $Q$, and the set of correct moves from $Q$. Only $\mathcal{E}$ can move $\operatorname{bck}(i)$, that is, only $\mathcal{E}$ can "backtrack" to the judgement number $i$ of $Q$, with the further limitation that if $i$ is the index of a positive judgement, this judgement has to be the last positive judgement of $Q$. Since $\mathcal{E}$ can come back to the logical move number $i$, the last logical move for $\mathcal{E}$ is not, necessarily, the last logical move of the sequence. We call the last logical move for $\mathcal{E}$ : the active move of the position. For each position $Q$ we define: the backtracking indexes, the active move and its index, the next player moving and its correct moves.

Definition 9. (Correct moves) Let $P$ be any position, having $n>0$ logical moves.

1. $i$ is a backtracking index of $P$, a bck-index for short, if: : (i) $1 \leq i \leq$ $n$; (ii) if the $i$-th judgement is positive, then the $i$-th judgement is the last positive judgement of $P$.

2. If the last move of $P$ is $s A$, the active move of $P$ is $s A$, of index $n$.

3. If the last move of $P$ is $\mathrm{bck}(i)$, the active move of $P$ is the logical move of index $i$ of $P$ if $i$ is a bck-index of $P$, the logical move of index $n$ o.w..

4. If the active move of $P$ is a disjunctive judgement, the player moving from $P$ is $\mathcal{E}$.

5. If the active move of $P$ is a conjunctive judgement, the player moving from $P$ is $\mathcal{A}$.

6. If $p$ is the player moving from $P$, and $s A$ the active move, the correct moves of $p$ are:

- drop (to drop out from the game)

- any $s^{\prime} A^{\prime}<_{1} s A$,

- if $p=\mathcal{E}$, also $\operatorname{bck}(i)$, for any $i$ bck-index of $P$. 
7. $Q$ is a correct extension of $P$ if $Q \geq P$, and for any $P \leq R @\langle m\rangle \leq$ $Q$, the move $m$ is correct from $R$.

If the active move of $P$ is t. $A$, we say that $\mathcal{E}$ defends, in $P$, the thesis $A$, while some Abelard attacks the thesis $A$. If the active move of $P$ is $\mathrm{f} . A$, we say that $\mathcal{E}$ attacks, in $P$, the thesis $A$ while some Abelard defends the thesis $A$. We now define the winner and the loser for any (finished) play $P$.

Definition 10. (loser and winner of a play) Let $P$ be any play.

1. If $P=Q$, drop, and $p$ is the player moving from $Q$, then $p$ loses in $P$, and its opponent wins.

2. If $P$ is infinite, then $\mathcal{E}$ loses in $P$, and $\mathcal{A}$ wins.

We allow a play to use any position $P$ as initial segment. The game associated $G_{P}$ to a position $P$ is the set of all positions we can reach from $P$ using only correct moves of the next player. Positions and sequents are interchangeable notions. Any position $P$ is associated to some sequent $\operatorname{seq}(P)=\langle\Gamma \vdash D, i\rangle$ (see the definition below). seq $(P)$ is defined by interpreting each move $m$ in $P$ as an operation building a sequent, by Def. 7.4. We imagine that, in the position $P, \mathcal{E}$ claims that $\langle\Gamma \vdash D, i\rangle$ is true, and $\mathcal{A}$ claims it is false. Conversely, any pointed sequent $\langle\Gamma \vdash D, i\rangle$ is associated to some canonical position $P=\operatorname{pos}(\Gamma \vdash D, i)$, and to some game $G(\Gamma \vdash D, i)$ having $P$ as initial position.

Definition 11. Assume $P=$ t. $A, m_{1}, \ldots, m_{k}$ is any position with $n>$ 0 logical moves. Let $\langle\Gamma \vdash D, i\rangle$ be any pointed sequent, with canonical indexing $\left\langle\Gamma^{\prime} \vdash D^{\prime}, i^{\prime}\right\rangle$, and $\Gamma=A_{1}, \ldots, A_{n}$.

1. The game $G_{P}$ associated to $P$ is the set of all correct extensions of $P$.

2. The sequent seq $(P)$ associated to $P$ is $\langle\emptyset \vdash A, 1\rangle+m_{1}+\ldots+m_{k}$ (see Def. 7.4).

3. The position $\operatorname{pos}(\Gamma \vdash D, i)$ associated to $\langle\Gamma \vdash D, i\rangle$ is: t. $D$, f. $A_{1}, \ldots$, f. $A_{n}$ if $i^{\prime}=n+1$, and $=$ t. $D$, f. $A_{1}, \ldots$, f. $A_{n}, \operatorname{bck}\left(i^{\prime}\right)$ if $1 \leq i^{\prime} \leq n$.

4. If $P=\operatorname{pos}(\Gamma \vdash D, i)$, then $G(\Gamma \vdash D, i)=G_{P}$. $G(D)=G(\vdash D, 1)$.

By definition unfolding, we can check that "taking the associated position" and "taking the associated sequent" are two operations inverse each other (up to index renaming).

Lemma 1. Let $\left\langle\Gamma^{\prime} \vdash D^{\prime}, i^{\prime}\right\rangle$ be the canonical indexing of the sequent $\langle\Gamma \vdash D, i\rangle$, and $P=\operatorname{pos}(\Gamma \vdash D, i)$ be the position associated to $\langle\Gamma \vdash$ $D, i\rangle$. Then $\operatorname{seq}(P)=\left\langle\Gamma^{\prime} \vdash D^{\prime}, i^{\prime}\right\rangle$ (i.e., "the sequent associated to the position associated to a sequent is the sequent itself", up to index renaming).

The initial position of the game $G(\Gamma \vdash D, i)$ is $P=\operatorname{pos}(\Gamma \vdash D, i)$. In the initial position of $G(\Gamma \vdash D, i), \mathcal{E}$ claims that $\operatorname{seq}(P)=\left\langle\Gamma^{\prime} \vdash D^{\prime}, i^{\prime}\right\rangle$ is true, while $\mathcal{A}$ claims it is false. The initial position of $G(A)$ is $\operatorname{pos}(\vdash$ $A, 1)=\mathrm{t} . A$. We will now define winning strategies for $\mathcal{E}$ on a game as particular recursive trees. We first define a coding for recursive trees, and an indexing Children $(x)$ for the children of a node $x$. 
Definition 12. (Coding recursive trees)

- A tree $T$ over $M$ is any set of finite lists over $M$, including the empty list \langle\rangle and closed under prefix.

- If $x \in T$, then Children $(x)=\{m \in M \mid x @\langle m\rangle \in T\}$ is an indexing for the children of a node $x$.

- A tree is recursive if it is coded by a recursive set.

- A labeling over a tree is any map $1: T \rightarrow I$ assigning some label $l(x) \in I$ to each $x \in T$.

From now on, we always code trees by sets of lists. If $x, y$ are lists, we denote the concatenation of $x, y$ by $x @ y$. We define winning strategies for $\mathcal{E}$ on a game $G_{P}$ as particular recursive well-founded trees $\sigma$ on the set Moves. Here is how $\sigma$ works. During the play, the current position of the play is always $P @ x$, for some $x \in \sigma . \sigma$ is defined in such a way that, whenever $\mathcal{E}$ moves from $P @ x$, there is exactly one child $x @\langle m\rangle \in \sigma$ of the current node $x \in \sigma . m \in$ Moves is the correct move $\neq$ drop suggested by $\sigma$ to $\mathcal{E}$ from $P @ x$. Since $m \in$ Moves, then $\mathcal{E}$ cannot play drop. Whenever $\mathcal{A}$ moves, instead, the children $y$ of $x$ are all $x @\langle m\rangle \in$ Moves which are correct moves $\neq$ drop of $\mathcal{A}$ from $P @ x$. $\mathcal{\mathcal { E }}$ chooses the child $x @\langle m\rangle$ corresponding to the actual move $m$ by $\mathcal{A}$ (unless $\mathcal{A}$ plays drop, in which case play ends). Since $\sigma$ is well-founded, after finitely many moves the play ends. The loser is necessarily $\mathcal{A}$, because $\mathcal{A}$ is the only player who can play drop.

Definition 13. (Recursive winning strategies for $\mathcal{E}$ on $G_{P}$ ) Fix any position $P$. Let $\sigma$ be any tree over the set Moves.

1. For any $x \in T$, we call $\operatorname{seq}_{P}(x)=\operatorname{seq}(P @ x)$ the sequent labeling $x$.

2. $\sigma$ is a strategy for $\mathcal{E}$ on $G_{P}$ if for all $x \in T$, if $p$ is the player moving from $P @ x$ :

- if $p=\mathcal{E}$, then Children $(x)=\{m\}$, for some correct move $m \neq$ drop from $P @ x$.

- if $p=\mathcal{A}$, then Children $(x)$ is the set of all correct moves $\neq \mathrm{drop}$ from $P @ x$.

3. $\sigma$ is winning if it is a well-founded tree. $\sigma$ is recursive if it is a recursive tree.

We can now define validity in our game semantics.

Definition 14. Let $\langle\Gamma \vdash D, i\rangle$ be any sequent of $\mathrm{L}_{\mathrm{G} 0}$.

1. $\sigma \models\langle\Gamma \vdash D$, iो if $\sigma$ is a recursive winning strategy for $\mathcal{E}$ on $G(\Gamma \vdash$ $D, i)$.

2. $\mathcal{G}=\langle\Gamma \vdash D$, iो if $\exists \sigma . \sigma|=\langle\Gamma \vdash D, i\rangle$.

3. $\mathcal{G} \models D$ if $\mathcal{G} \models\langle\vdash D, 1\rangle$.

We can characterize a strategy on any game $G_{P}$ as follows:

Lemma 2. $\sigma$ is a recursive winning strategy for $\mathcal{E}$ on $G_{P}$ if and only if $\sigma$ is a recursive well-founded tree, and, for all $x \in \sigma$, if the active move of $P @ x$ is sA, then:

- either $s A$ is disjunctive and Children $(x)=\{\operatorname{bck}(j)\}$, for some $j$ bckindex of $P @ x$, 
- or $s A$ is disjunctive and Children $(x)=\left\{s^{\prime} A^{\prime}\right\}$, for some $s^{\prime} A^{\prime}<_{1} s A$,

- or $s A$ is conjunctive and Children $(x)=\left\{s^{\prime} A^{\prime} \mid s^{\prime} A^{\prime}<_{1} s A\right\}$.

Proof. By unfolding Def. 13, 9.

In $\S 8$ we will prove that for all $D \in \mathrm{L}_{\text {HAO }}$ we have $\mathcal{G} \models D$ if and only if $D$ is a theorem of $\mathrm{HA}_{\mathrm{inf}}$, Infinitary Intuitionistic Arithmetic with $\omega$-rule.

\section{Unfolding our definition of game}

In this section we informally describe the games $G(A)$ and $G(\Gamma \vdash D, i)$, by unfolding the formal definition from the previous section. We stress the differences with Lorentzen's games. which are: our games extend Tarski games ( $\S 4.1)$, are defined by restricting Coquand's notion of backtracking $(\S 4.2,4.3)$, and make a reduced use of insertion of dummy moves in the play (§4.4). In $\S 10$ we will include an example of winning strategy and one example of play.

\subsection{Interpreting positive formulas}

Let $A \in \mathrm{L}_{0}^{+}$be any positive formula (i.e., any closed formula in the connectives $F, T, \vee, \wedge, \exists, \forall)$. Let $P \in G(A)$. We claim that $P$ runs as in Tarski play [9]. According to Def. 11, the initial position of $G(A)$ is t. $A$. The sub-judgements of t. $A$ are all t. $B$, for $B$ sub-formula of $A$. t. $A$ has no negative sub-judgements $\mathrm{f} . B$, because there are no $\rightarrow, \Rightarrow, \neg$ in $A$. By Def. $9, \mathcal{A}$ cannot backtrack, while $\mathcal{E}$ can only backtrack to the last positive judgement, hence to the last judgement, because all judgements in $P$ are positive. Thus, the active move of any non-empty prefix of $P$ is always the last judgement. Therefore all logical move are some immediate sub-judgement of the previous judgement. This means we can skip all moves $\operatorname{bck}(i)$ from $P$, and we still obtain a legal play. After we simplify $P$ in this way, we have $P=$ t. $A_{1}$, t. $A_{2}$, t. $A_{3}, \ldots$, t. $A_{n}$, for some $A=A_{1}>_{1} A_{2}>_{1} \ldots>_{1} A_{n}$. Correct moves are exactly as in Tarski games [9]. In Lorentzen, the relation with this simple and basic notion of game is instead somehow hidden. If t. $A_{i}$ is a disjunctive formula, then $\mathcal{E}$ chooses t. $A_{i+1}$, and if t. $A_{i}$ is a conjunctive formula, then $\mathcal{A}$ chooses t. $A_{i+1}$. In all moves t. $A_{i}, \mathcal{E}$ defends $A_{i}$ while $\mathcal{A}$ attacks $A_{i}$. This play has a single "thread", which is a debate about the truth of $A$.

More in detail, if $A_{i}=B_{1} \vee B_{2}, \exists x . B[x]$, then $\mathcal{E}$ moves next. Her correct moves (apart from drop) are in the first case t. $A_{i+1}=$ t. $B_{i}$ for some $i=1,2$, in the second one, t. $A_{i+1}=$ t. $B[t]$ for some closed term $t . \mathcal{E}$ defends a disjunctive formula $A_{i}$ by defending some $A_{i+1}<_{1} A_{i}$. If she can convince $\mathcal{A}$ that $A_{i+1}$ is true, she will also convince $\mathcal{A}$ that $A_{i}$ is true. If $A_{i}=B_{1} \wedge B_{2}, \forall x . B[x]$, then $\mathcal{A}$ moves next. His correct moves (apart from drop) are in the first case t. $A_{i+1}=$ t. $B_{i}$ for some $i=1,2$, in the second one, t. $A_{i+1}=\mathrm{t} . B[t]$ for some closed term $t$. $\mathcal{A}$ attacks a conjunctive formula $A_{i}$ by attacking some $A_{i+1}<_{1} A_{i}$. If he can convince $\mathcal{E}$ that $A_{i+1}$ is false, he will also convince $\mathcal{E}$ that $A_{i}$ is false. 
If $A_{i}=a$ is atomic closed, then $\mathcal{E}$ moves next. Her only move (apart from drop) is $T$ if $a$ is true, and $F$ if $a$ is false. If $A_{i}=F, T$, then the only move from $A_{i}$ is drop, hence $i=n$ is the last index of the play. Since there is no backtracking, $A_{i}$ is a losing position for the next player. Indeed, if $A_{i}=F$, then $\mathcal{E}$ moves next, she moves drop and she loses. $\mathcal{E}$ fails defending $F: \mathcal{A}$ convinces $\mathcal{E}$ that $F$ is false. If $A_{i}=T$, then $\mathcal{A}$ moves next, he moves drop and he loses. $\mathcal{A}$ fails attacking $T: \mathcal{E}$ convinces $\mathcal{A}$ that $T$ is true.

In Tarski games, the roles of $\mathcal{E}$ and $\mathcal{A}$ are perfectly symmetrical, and $A$ is true if and only if $\mathcal{E}$ has a winning strategy on the game associated to $A$. This is not the case in our game interpretation, because we only allow recursive strategies for $\mathcal{E}$, while there is no restriction on the strategy for $\mathcal{A}$. $\mathcal{E}$ is a finite and fallible being, while all Abelard's in the array $\mathcal{A}$ can be omniscient. The game associated to $A$ is therefore strongly biased in favor of $\mathcal{A}$. Indeed, $\mathcal{E}$ has no recursive winning strategy on the game associated to some true $A \in \mathrm{L}_{0}^{+}$(see Corollary 2.1). The canonical strategy for the Abelard's is selecting some false sub-judgement if any exists, otherwise drop out. This strategy is winning if and only if $A$ is true.

\subsection{Interpreting the negation of a positive formula}

Let $A \in \mathrm{L}_{0}^{+}$be any positive formula. We discuss now the game $G(\mathbf{A} \vdash$ $F$ ) associated to $\mathbf{A} \vdash F$ (a sequent with active formula $A$ ). $\mathbf{A} \vdash F$ is equivalent to the negation of $A$. The initial position of the game is t.F,f.A. By Def. 11, sub-judgments of f. $A$ are exactly all f. $B$ with $B$ subformula of $A$ : all sub-judgements of $\mathrm{f} . A$ have sign $\mathrm{f}$., because there is no connective $\rightarrow, \Rightarrow, \neg$ in $A$. In all judgements $\mathrm{f} . B, \mathcal{A}$ defends $B$ while $\mathcal{E}$ attacks $B$. There is some symmetry with the game $G(A)$, but it is only apparent. Here, backtracking is a useful move for $\mathcal{E}$, because she can come back to any previous logical move f.B (there is no restriction for backtracking on negative judgements). This means that, whenever $\mathcal{E}$ thinks she did a mistake by selecting some f. $B^{\prime}<_{1}$ f. $B$, she can come back to f. $B$ and select a different f. $B^{\prime \prime}<_{1}$ f. $B$. This starts a new "thread" in the play, a debate about the falsity of $f . B^{\prime \prime}$. The play from f. $B$ evolves as it were a Coquand's play [5]. Initially, there is only one "thread" in the play, a debate about the falsity of $A$. Each time $\mathcal{E}$ backtracks, some thread forks, and a new thread starts.

We interpret the fact that $\mathcal{A}$ cannot backtrack by imagining that there is some Abelard from the array $\mathcal{A}$ associated to each negative judgement, who ignores the history of the play, and moves using this judgement as only information. Whenever $\mathcal{E}$ moves f. $B^{\prime}$ from f.B, a new Abelard is detached from the array $\mathcal{A}$, in order to defend $B$. This Abelard waits, possibly forever, that $\mathcal{E}$ comes back to f. $B$. If and when this happens, $\mathcal{E}$ moves $f . B^{\prime \prime}$ against this Abelard, opening a new thread in the play, while again, a new Abelard is detached in order to defend $B$. The number of Abelard's in the play grows with time. At each step, there are as many Abelard's as negative judgements in $P . \mathcal{E}$ is only facing one of them at the time, the one defending the active judgement of $P$. $\mathcal{E}$ can switch at any time to a different Abelard, moving bck $(i)$ for some $i$. 
We interpret the fact that $\mathcal{E}$ can change a wrong move, and therefore knows the history of the play, by the fact that $\mathcal{E}$ is a single individual. Through backtracking, this individual evolves with time, "learning from her mistakes". Each Abelard in the array $\mathcal{A}$ does not know the history of the game, and he cannot backtrack. Therefore he must choose the best move on the first try, using only the information available from the judgement. This is not a problem for the Abelard's, because we assumed all of them to be omniscient.

The fact that $\mathcal{E}$ can learn from her mistake compensates for the omniscience of $\mathcal{A}$. In fact (assuming that $A$ is a positive formula), the game $G(\mathbf{A} \vdash F)$ is perfectly equilibrate: $\mathcal{E}$ has a recursive winning strategy if and only if $A$ is false in the standard model $N$ (Corollary 2.2). The canonical strategy for the Abelard's, select some false sub-judgement if any exists, otherwise drop out, is winning if and only if $A$ is true. For some $A$ there is no recursive winning strategy for the Abelard's: in this case, the omniscience of the Abelard's is essential to make the game equilibrate.

There are two asymmetries in our notion of game. There is an asymmetry $\mathcal{E} / \mathcal{A}$, and there is an asymmetry defence/attack. A move defending a formula, done by any player, must be perfect on the first try: truth must be flawless. A move attacking a formula can be retracted a finite number of times: falsity can be established after finitely many attempts. Only the weaker player, $\mathcal{E}$, has the privilege of retracting moves. $\mathcal{A}$ has already the advantage of omniscience (i.e., of possibly using non-recursive strategies). Therefore $\mathcal{A}$ cannot retract moves.

An obvious generalization of the game $G(\mathbf{A} \vdash F)$ is the game $G\left(A_{1}, \ldots, A_{n} \vdash\right.$ $F)$, for $A_{1}, \ldots, A_{n} \in \mathrm{L}_{0}^{+}$. The game runs as the game $G(\mathbf{A} \vdash F)$, except that, already in the initial position, there are $n$ negative judgment, therefore $n$ Abelard's against $\mathcal{E}$, and $n$ threads in the play. In the initial position, $\mathcal{E}$ is facing the Abelard defending f. $A_{n}$, but she can change the Abelard she is facing at will.

\subsection{Interpreting the entailment relation $A_{1}, \ldots, A_{n} \vdash D$}

Let $A_{1}, \ldots, A_{n}, D \in \mathrm{L}_{0}^{+}$. The initial position of $G\left(A_{1}, \ldots, A_{n} \vdash D\right)$ is, according to our definition, t. $D, \mathrm{f} . A_{1}, \ldots, \mathrm{f} . A_{n}$. This game is a combination of the games discussed in the two previous subsections. There are $n+1$ Abelard's against $\mathcal{E}$, one attacking $D$, and $n$ more Abelard's defending $A_{1}, \ldots, A_{n}$. Hayashi proposed to call "tutors" these latter, because $\mathcal{E}$ hopes to learn from them how to defend $D$. By definition unfolding, $\mathcal{E}$ is currently facing the Abelard defending $A_{n} . \mathcal{E}$ can backtrack to any negative judgement, but only to the last positive judgement. This means that $\mathcal{E}$ can learn by trial-and-error the best move to attack each $A_{1}$, while her move defending $D$ is irreversible, and must be correct on the first try, and this fact makes her weaker. The thread of the play from t. $D$ evolves as it were a Tarski play with a recursive strategy (see $\S 4.1$ ), while the threads from all $A_{i}$ evolve as they were Coquand's play [5] (see $\S 4.2)$. The threads from all $A_{i}$ can fork and grow in number with time. $\mathcal{E}$ is bounded to use a recursive strategy. This fact, combined with the fact that her moves from $D$ must be correct on the first try, makes the 
game strongly biased in favor of $\mathcal{A}$. In fact, $\mathcal{E}$ has no recursive winning strategy on $G\left(A_{1}, \ldots, A_{n} \vdash D\right)$, for some true sequent $A_{1}, \ldots, A_{n} \vdash D$ (see Corollary 2.1).

\subsection{Interpreting the intuitionistic implication $\Rightarrow$}

We now consider the most general case, a game $G\left(A_{1}, \ldots, A_{n} \vdash D, i\right)$, for some $A_{1}, \ldots, A_{n}, D \in \mathrm{L}_{\mathrm{G} 0}$. There are four new cases: when the active move of $P$ is t. $C$ or f. $C$, for $C=A \rightarrow B$ or $A \Rightarrow B$. $\rightarrow$ is an operator defining games, and is the standard interpretation of implication in Tarski games, but not in our games. In our game semantics, we interpret the intuitionistic implication as $\Rightarrow$.

If the active move of $P$ is t. $A \rightarrow B$, then $\mathcal{E}$ moves next. She can move f. $A$, then she can backtrack to t. $A \rightarrow B$, and move t. $B$. In this way the thread of the play from t. $A \rightarrow B$ consists of two threads evolving in parallel, one from $\mathrm{f} . A$, the other from $\mathrm{t}$. $A$. The thread from $\mathrm{f} . A$ evolves like a Coquand's play, the thread from t. $B$, as a Tarski play (see $\S 4.2$, $4.1)$.

The case in which the active move of $P$ is t. $A \Rightarrow B$ is almost the same. $\mathcal{A}$ moves from t. $A \Rightarrow B$ to t. $A \rightarrow B$, then the play continues as we just sketched.

Assume the active move of $P$ is $\mathrm{f} . A \rightarrow B$. Then $\mathcal{A}$ moves next, and according if he moves t. $A$ or $\mathrm{f} . B$, the thread of the play from $\mathrm{f} . A \rightarrow B$ continues from t. $A$, or from f.B.

The only non-trivial case is when the active move of $P$ is $\mathrm{f} . A \Rightarrow B$. This position is a disjunctive judgement because $A \Rightarrow B$ is conjunctive. $\mathcal{E}$ moves next, and her correct moves are either $\mathrm{f} . A \rightarrow B$, or $\operatorname{bck}(i)$ for some $i$. Thus, by adding the dummy node f. $A \Rightarrow B$ as father of $\mathrm{f} . A \rightarrow B$, we allow to $\mathcal{E}$ more chances to backtrack. Backtracking is a powerful move for $\mathcal{E}$, therefore adding the node $A \Rightarrow B$ makes $\mathcal{E}$ stronger. Indeed, $\Rightarrow$ is a sound and complete interpretation of intuitionistic implication ( $(8)$, while $\rightarrow$ is not $(\S 5)$.

In game theoretical terms, any judgement f. $A \Rightarrow B$ is a node in which $\mathcal{E}$ can (thanks to backtracking) delay the reply by Abelard to the moment most suitable for her. This possibility of producing a delay makes the difference (sometimes, a huge difference) between game interpretation of $\Rightarrow$ and $\rightarrow$. The difference between $\Rightarrow$ and $\rightarrow$ cannot be expressed in logical terms, this is why we consider $\rightarrow$ only as an operator in the language $\mathrm{L}_{\mathrm{G}}$ denoting games, and not as a logical connective.

In Lorentzen's games, the trick of interleaving a dummy move of one player before a real move of the other player is used for $\vee, \exists$. In [6], p.116, the player $Y$ attacking a disjunction $A_{1} \vee A_{2}$ performs a dummy move called $\vee$. Thanks to this apparently useless move, the player $X$ defending $A_{1} \vee A_{2}$ can either answer $A_{i}$ for some $i=1,2$, or backtrack to some previous move. A similar situation happens for $\exists x . A[x]$. In HylandOng's interpretation of implication there is a similar situation: Opponent asks for a value of an arrow type, and Player can either answers to this question or backtrack. Lorentzen, instead, interpreted implication through a different trick: in order to defend $A \Rightarrow B$, you do not have to choose between attacking $A$ or defending $B$, but you have to do both in 
parallel. We simplified this pattern, making all moves the choice of a subjudgement, and interleaving a dummy move only to interpret implication. We cannot avoid dummy moves completely, but we localized the source of this problem in the implication connective alone.

\section{The operator $\rightarrow$ is not a sound and complete interpretation of Intuitionistic implication}

In this section we check that we cannot interpret intuitionistic implication with just $\rightarrow$. The correct interpretation is instead $\Rightarrow(\S 8)$.

Lemma 3. Let $A(x), C(x) \in \mathrm{L}_{\mathrm{HAO}}$.

1. If $\mathcal{G} \models \forall x .((A(x) \rightarrow F) \rightarrow C(x))$, then $\mathcal{G} \models \forall x . \neg(A(x) \rightarrow F) \vee C(x)$.

2. $\rightarrow$ is not a sound and complete interpretation of intuitionistic implication.

\section{Proof.}

1. Assume $\mathcal{E}$ has a recursive winning strategy $\sigma$ on the game with initial position t. $\forall x .((A(x) \rightarrow F) \rightarrow C(x))$. We have to define, recursively over on $t$, a recursive winning strategy $\tau$ for $\mathcal{E}$, either on $G(\neg(A(t) \rightarrow$ $F)$ ), or on $G(C(t))$. Any play over t. $\forall x .((A(x) \rightarrow F) \rightarrow C(x))$ starts with a move t. $(A(t) \rightarrow F) \rightarrow C(t)$ by $\mathcal{A}$, for some closed term $t$. Let $P=$ t. $\forall x .((A(x) \rightarrow F) \rightarrow C(x))$, t. $(A(t) \rightarrow F) \rightarrow C(t) \in \sigma$ be the current position of the play. The player moving from $P$ is $\mathcal{E}$. Then, by definition of strategy, $P$ has a unique child in $\sigma$.

- Assume P, t. $C(t)$ is the only child of $P$ in $\sigma . P$, t. $C(t)$ has last positive judgement t. $C(t)$. $\mathcal{E}$, from now on, cannot backtrack to any judgement in $P$ (both judgements in $P$ are positive). Let $\rho=\{Q \in \sigma \mid Q \geq P$, t. $C(t)\}$. $\rho$ is a recursive winning strategy for $\mathcal{E}$ on $G_{P, \mathrm{t} . C(t)}$, including only moves bck $(i)$ with $i>2$, because $\rho$ never backtracks to $P$. Remove the prefix $P$ from any $Q \in \rho$, and replace each $\operatorname{bck}(i)$ in $Q$ with $\operatorname{bck}(i-2)$. The resulting tree $\tau$ is a strategy on $G_{\mathrm{t} . C(t)}=G(C(t))$, winning because $\rho$ is.

- Assume $P$, f. $A(t) \rightarrow F$ is the only child of $P$ in $\sigma$. If $\mathcal{A}$ replies t. $A(t)$, then the current position is $P, \mathrm{f} . A(t) \rightarrow F, \mathrm{t} . A(t)$, and t. $A(t)$ is the last positive judgement. $\mathcal{E}$, from now on, cannot backtrack to any judgement in $P$ (both judgements in $P$ are positive). Let $\rho=\{Q \in \sigma \mid Q \geq P\}$. $\rho$ is a recursive winning strategy for $\mathcal{E}$ on $G_{P}$, including only moves bck $(i)$ with $i>2$, because $\rho$ never backtracks to $P$. Replace $P$ with t. $\neg(A(t) \rightarrow F)$ in any $Q \in \rho$, and replace each $\operatorname{bck}(i)$ in $Q$ with $\operatorname{bck}(i-1)$. The resulting tree $\tau$ is a strategy on for $\mathcal{E}$ on $G_{\mathrm{t} . \neg(A(t) \rightarrow F)}=$ $G(\neg(A(t) \rightarrow F))$, because f. $A(t) \rightarrow F$, the first move suggested by $\rho$ from $P$, is a correct move also from t. $\neg(A(t) \rightarrow F)$. $\tau$ is winning because $\rho$ is.

2. Assume, for contradiction, that $\rightarrow$ is a sound and complete interpretation of intuitionistic implication. Then the set of intuitionistic theorems is closed under the rule expressed by the previous point. For contradiction, consider the Introduction schema for $\neg: \forall x .((A(x) \rightarrow$ 
$F) \rightarrow \neg A(x))$. Apply point 1 and deduce that $\forall x . \neg(A(x) \rightarrow F) \vee$ $\neg A(x)$ is an intuitionistic theorem. But this schema, called Weak Excluded Middle, is not an intuitionistic theorem if $A(x) \in \mathrm{L}_{\mathrm{HAO}}$ is not a recursive predicate.

\section{$6 \quad \mathrm{HA}_{\mathrm{inf}}$, Intuitionistic Arithmetic with $\omega$-rule}

In this section we introduce $\mathrm{HA}_{\text {inf }}$, an infinitary sequent calculus (with pointed sequents) for Intuitionistic Arithmetic. We also introduce an infinitary logic $\mathcal{G}_{\text {inf }}$ for deriving validity of formulas of $\mathrm{L}_{\mathrm{G} 0}$ in our game semantics. Proofs in $\mathcal{G}_{\text {inf }}$ are isomorphic to winning strategies (Theorem 2 ). Eventually, in $\S 8$ we prove that $\mathcal{G}_{\text {inf }}$ is a conservative extension of $\mathrm{HA}_{\text {inf }}$, and we conclude that our game semantics is sound and complete for $\mathrm{HA}_{\text {inf }}$.

The language of $\mathrm{HA}_{\mathrm{inf}}$ is $\mathrm{L}_{\mathrm{HAO}}$ and the language for $\mathcal{G}_{\text {inf }}$ is $\mathrm{L}_{\mathrm{GO}}$. We first define logical rules of $\mathcal{G}_{\text {inf }}$ and $\mathrm{HA}_{\mathrm{inf}}$ a synthetic way, using the operation $\langle\Gamma \vdash D, i\rangle+s A$ from Def. 7 . Then we unfold our definition, in order to check that it is equivalent to the usual one for $\mathrm{HA}_{\mathrm{inf}}$.

Definition 15. - (Logical Rules for $\mathrm{HA}_{\text {inf }}$ ) Assume $\langle\Gamma \vdash D, i\rangle$ is a sequent of $\mathrm{L}_{\mathrm{HAO}}$, with active formula $A$ of sign $s$. Let $s^{\prime} A^{\prime}<_{1} s A$ in $\mathrm{L}_{\mathrm{HAO}}$. Then a logical rule of conclusion $\langle\Gamma \vdash D, i\rangle$ has premise one $\langle\Gamma \vdash D, i\rangle+s^{\prime} A^{\prime}$ if $s A$ is disjunctive, and all $\langle\Gamma \vdash D, i\rangle+s^{\prime} A^{\prime}$ if $s A$ is conjunctive.

- (Logical Rules for $\left.\mathcal{G}_{\text {inf }}\right)$. The definition is obtained from the definition for $\mathrm{HA}_{\text {inf }}$, by replacing the language $\mathrm{L}_{\mathrm{HAO}}$ with the language $\mathrm{L}_{\mathrm{GO}}$.

In $\mathcal{G}_{\text {inf }}$, for each sequent there is (at most) one logical rule having conclusion this sequent. In the definition above, there are 11 possible cases for $A$ : $A$ true or false atomic formula, or $A$ starting any of the 9 connectives of $\mathrm{L}_{\mathrm{G}}$. There are 2 possible signs. Therefore there are at most $11 \times 2=22$ possible cases for logical rules. There is no logical rule, however, if $s A$ is disjunctive and there is no $s^{\prime} A^{\prime}<_{1} s A$, because a logical rule for a disjunctive $s A$ requires one $s^{\prime} A^{\prime}<_{1} s A$. The are only two cases of this kind: $s A=$ t.F, f.T, both disjunctive and without immediate sub-judgements. Therefore, if we unfold the definition of logical rule, we obtain $22-2=20$ cases for a logical rule in $\mathcal{G}_{\text {inf }}{ }^{1}$ :

Definition 16. (Logical Rules for $\mathcal{G}_{\text {inf }}$ ) We write the active occurrence of any sequent boldface.

- (Rules for $F, T)$

$$
\overline{\Gamma, \mathbf{F} \vdash D} \quad \overline{\Gamma \vdash \mathbf{T}}
$$

- (Atomic logical rules) For any a atomic false, and any $b$ atomic true (true and false in the standard model $N$ ):

$$
\frac{\Gamma, a, \mathbf{F} \vdash D}{\Gamma, \mathbf{a} \vdash D} \quad \frac{\Gamma \vdash \mathbf{F}}{\Gamma \vdash \mathbf{a}} \quad \frac{\Gamma, b, \mathbf{T} \vdash D}{\Gamma, \mathbf{b} \vdash D} \quad \frac{\Gamma \vdash \mathbf{T}}{\Gamma \vdash \mathbf{b}}
$$

${ }^{1}$ In Def. 16 there are, in fact, 21 different patterns, because there are two patterns in the case $A$ is an implication $B \rightarrow C$, and $A$ is in the right-hand-side of a sequent. 
- (Conjunctive logical rules for $\wedge, \forall, \vee, \exists$ )

$$
\begin{gathered}
\frac{\Gamma \vdash \mathbf{C}_{\mathbf{i}}(\text { for } i=1,2)}{\Gamma \vdash \mathbf{C}_{\mathbf{1}} \wedge \mathbf{C}_{\mathbf{2}}} \quad \frac{\Gamma \vdash \mathbf{A}[\mathbf{t}] \text { (for all closed terms } t)}{\Gamma \vdash \forall \mathbf{x} . \mathbf{A}} \\
\frac{\Gamma, C_{1} \vee C_{2}, \mathbf{C}_{\mathbf{i}} \vdash D(\text { for } i=1,2)}{\Gamma, \mathbf{C}_{\mathbf{1}} \vee \mathbf{C}_{\mathbf{2}} \vdash D} \frac{\Gamma, \exists x . A, \mathbf{A}[\mathbf{t}] \vdash D(\text { for all closed terms } t)}{\Gamma, \exists \mathbf{x} . \mathbf{A} \vdash D}
\end{gathered}
$$

- (Disjunctive logical rules for $\wedge, \forall, \vee, \exists$ ). Let $i=1$ or $i=2$, and $t$ be any closed term.

$$
\begin{array}{cl}
\frac{\Gamma \vdash \mathbf{C}_{\mathbf{i}}}{\Gamma \vdash \mathbf{C}_{\mathbf{1}} \vee \mathbf{C}_{\mathbf{2}}} & \frac{\Gamma \vdash \mathbf{A}[\mathbf{t}]}{\Gamma \vdash \exists \mathbf{x} \cdot \mathbf{A}} \\
\frac{\Gamma, C_{1} \wedge C_{2}, \mathbf{C}_{\mathbf{i}} \vdash D}{\Gamma, \mathbf{C}_{\mathbf{1}} \wedge \mathbf{C}_{\mathbf{2}} \vdash D} & \frac{\Gamma, \forall x \cdot A, \mathbf{A}[\mathbf{t}] \vdash D}{\Gamma, \forall \mathbf{x} \cdot \mathbf{A} \vdash D}
\end{array}
$$

- (Logical rules of implication $\rightarrow$ ) Remark that the logical rule for $A \rightarrow B$ in the right-hand-side has two sub-cases.

$$
\begin{gathered}
\frac{\Gamma, \mathbf{A} \vdash A \rightarrow B}{\Gamma \vdash \mathbf{A} \rightarrow \mathbf{B}} \quad \frac{\Gamma \vdash \mathbf{B}}{\Gamma \vdash \mathbf{A} \rightarrow \mathbf{B}} \quad \frac{\Gamma, A \rightarrow B \vdash \mathbf{A} \quad \Gamma, A \rightarrow B, \mathbf{B} \vdash D}{\Gamma, \mathbf{A} \rightarrow \mathbf{B} \vdash D} \\
-(\text { Logical rules of intuitionistic implication } \Rightarrow) \\
\frac{\Gamma \vdash \mathbf{A} \rightarrow \mathbf{B}}{\Gamma \vdash \mathbf{A} \Rightarrow \mathbf{B}} \quad \frac{\Gamma, A \Rightarrow B, \mathbf{A} \rightarrow \mathbf{B} \vdash D}{\Gamma, \mathbf{A} \Rightarrow \mathbf{B} \vdash D}
\end{gathered}
$$

- (Logical rules of negation)

$$
\frac{\Gamma, \mathbf{A} \vdash \neg A}{\Gamma \vdash \neg \mathbf{A}} \quad \frac{\Gamma, \neg A \vdash \mathbf{A}}{\Gamma, \neg \mathbf{A} \vdash D}
$$

We can unfold the logical rules for $\mathrm{HA}_{\mathrm{inf}}$ in a similar way. The only difference between $\mathrm{HA}_{\text {inf }}$ and $\mathcal{G}_{\text {inf }}$ is that in $\mathrm{HA}_{\mathrm{inf}}$ the rules for $A \rightarrow B$ are skipped, and the rules for $A \Rightarrow B$ have hypotheses with active formulas $A, B$, because the immediate subformulas of $A \Rightarrow B$ in $\mathrm{L}_{\mathrm{HA}}$ are $A, B$ :

$$
\frac{\Gamma, \mathbf{A} \vdash A \Rightarrow B}{\Gamma \vdash \mathbf{A} \Rightarrow \mathbf{B}} \quad \frac{\Gamma \vdash \mathbf{B}}{\Gamma \vdash \mathbf{A} \Rightarrow \mathbf{B}} \quad \frac{\Gamma, A \Rightarrow B \vdash \mathbf{A} \quad \Gamma, A \Rightarrow B, \mathbf{B} \vdash D}{\Gamma, \mathbf{A} \Rightarrow \mathbf{B} \vdash D}
$$

We consider only one structural rule both for $\mathrm{HA}_{\text {inf }}$ and $\mathcal{G}_{\text {inf }}$, Exchange, switching the active formula of a sequent.

Definition 17. (Structural Rule for $\mathrm{HA}_{\text {inf }}$ and $\mathcal{G}_{\text {inf }}$ : Exchange) Let $\Gamma \vdash D$ be any sequent, and $i, j$ any two occurrences of formulas of $\Gamma \vdash D$.

- The Exchange rule, or E-rule for short, is the following: derive $\langle\Gamma \vdash$ $D, j\rangle$ from $\langle\Gamma \vdash D, j\rangle+\operatorname{bck}(i)$.

- DisjE, ConjE, ConjE $\rightarrow$-rules are the restriction of the E-rule, when the occurrence $j$ is, respectively: disjunctive, conjunctive, conjunctive and equal to $B_{1} \rightarrow B_{2}$ for some $B_{1}, B_{2}$.

- The Exchange rules for $\mathrm{HA}_{\text {inf }}, \mathcal{G}_{\text {inf }}$ are $E$ and DisjE.

By unfolding the definition of $\langle\Gamma \vdash D, j\rangle+\operatorname{bck}(i)$, we can write the E-rule as follows: for any two occurrences $i, j$ of $\Gamma \vdash D$, 


$$
\frac{\langle\Gamma \vdash D, i\rangle}{\langle\Gamma \vdash D, j\rangle}
$$

E-rule says that two sequents differing only for the active formula are equivalent. In Lemma 6.5, we will prove that ConjE is conditionally derivable in $\mathcal{G}_{\text {inf }}$ from DisjE for all sequents of $\mathrm{L}_{\mathrm{HA}}$ (i.e., for all sequents without $\rightarrow)$. We will deduce that $\mathcal{G}_{\text {inf }}$ is a conservative extension of $\mathrm{HA}_{\text {inf }}$. Besides, strategies in our game semantics and proofs of $\mathcal{G}_{\text {inf }}$ can be identified (see Theorem 2). By combining the two remarks, we will conclude that our game semantics are sound and complete for $\mathrm{HA}_{\mathrm{inf}}$.

Proofs of $\mathrm{HA}_{\mathrm{inf}}$ ( of $\mathcal{G}_{\mathrm{inf}}$ ) are all well-founded recursive trees, labeled with sequents, and such that the sequent labeling each node is the conclusion of some rule of $\mathrm{HA}_{\mathrm{inf}}$ ( of $\mathcal{G}_{\mathrm{inf}}$ ). We code proofs as trees over Moves, in order to stress the similarity between proofs of $\mathcal{G}_{\text {inf }}$ and winning strategies.

Definition 18. Fix any sequent $S=\langle\Gamma \vdash D, i\rangle$ of $\mathrm{L}_{\mathrm{GO}}$ (of $\mathrm{L}_{\mathrm{HAO}}$ ). Assume $\Pi$ is any well-founded, recursive tree over Moves.

1. The labeling of $\Pi$ with sequents is, for all $x=\left\langle m_{1}, \ldots, m_{k}\right\rangle \in \Pi$ : $\operatorname{seq}_{S}(x)=S+m_{1}+\ldots+m_{k}$ (see Def. 7.4).

2. $\Pi$ is a proof of $S$ in $\mathrm{HA}_{\text {inf }}$ (in $\mathcal{G}_{\text {inf }}$ ) if for all $x \in \Pi$, there is some rule of $\mathrm{HA}_{\text {inf }}$ (in $\mathcal{G}_{\text {inf }}$ ) whose conclusion is: $\operatorname{seq}_{S}(x)$, and whose assumptions are: $\left\{\mathbf{s e q}_{S}(x @\langle m\rangle) \mid x @\langle m\rangle \in \Pi\right\}$.

3. $S$ is provable in $\mathrm{HA}_{\text {inf }}$ (in $\mathcal{G}_{\text {inf }}$ ) if there is some proof $\Pi$ of $S$ in $\mathrm{HA}_{\text {inf }}$ (in $\mathcal{G}_{\text {inf }}$ ).

We unfold the definition above, in order to explain how we code each rule of $\mathrm{HA}_{\mathrm{inf}}$ and $\mathcal{G}_{\mathrm{inf}}$. The root of $\Pi$ is coded \langle\rangle . Assume $x \in \Pi$, and the sequent $\operatorname{seq}(x)$ labeling $x$ has active move $s A$. If $x$ is the conclusion of some logical rule, then the children of $x$ in $\Pi$ are coded by all (by some) $x @\left\langle s^{\prime} A^{\prime}\right\rangle$, for $s^{\prime} A^{\prime}<_{1} s A$, according if $x$ is conjunctive or disjunctive. If $x$ is the conclusion of some structural rule, then the children of $x$ in $\Pi$ are coded by some $x @\langle\operatorname{bck}(i)\rangle$.

Introduction rule for $\rightarrow$ from Natural Deduction (if $\Gamma, A \vdash \mathbf{B}$, then $\Gamma \vdash$ $\mathbf{A} \rightarrow \mathbf{B}$ ) is conditionally derivable in $\mathrm{HA}_{\mathrm{inf}}{ }^{2}$. All other structural rules are derivable in $\mathrm{L}_{\mathrm{HA}}$ : identity, weakening, exchange (for all formulas), contraction in the left-hand side and cut. For instance, all previous rules in the left-hand side implicitly include contraction. Indeed, from $C_{1} \wedge$ $C_{2}, C_{1} \vdash D$ we infer $C_{1} \wedge C_{2} \vdash D$, instead of $C_{1} \wedge C_{2}, C_{1} \wedge C_{2} \vdash D$. We can characterize proofs of $\mathcal{G}_{\mathrm{inf}}$ as particular trees of moves.

Lemma 4. Let $\Pi$ be any well-founded recursive tree over Moves. Assume $S=\langle\Gamma \vdash D, i\rangle$. Then $\Pi$ is a proof of $S$ in $\mathcal{G}_{\text {inf }}$ if and only if, for all $x \in \Pi$, if the active formula $A$ of $\operatorname{seq}_{S}(x)$ has sign $s$, then:

- either $s A$ is disjunctive and Children $(x)=\{\operatorname{bck}(j)\}$, for some index $j$ of $\operatorname{seq}_{S}(x)$;

- or $s A$ is disjunctive and Children $(x)=\left\{s^{\prime} A^{\prime}\right\}$, for some $s^{\prime} A^{\prime}<_{1} s A$.

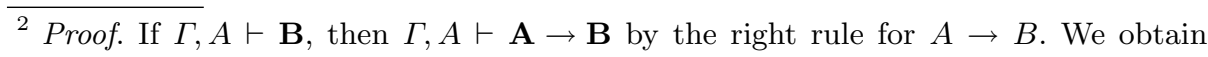
$\Gamma, \mathbf{A} \vdash A \rightarrow B$ by Exchange, and we conclude $\Gamma \vdash \mathbf{A} \rightarrow \mathbf{B}$ again by the right rule for $A \rightarrow B$. 
- or $s A$ is conjunctive and Children $(x)=\left\{s^{\prime} A^{\prime} \mid s^{\prime} A^{\prime}<_{1} s A\right\}$.

Proof. By definition unfolding, and because the only structural rule of $\mathcal{G}_{\text {inf }}$ is DisjE.

We will prove in Theorem 3 that $\mathcal{G}_{\text {inf }}$ is a conservative extension of $\mathrm{HA}_{\text {inf }}$. In $\S 3$, from this fact we will derive that our game semantic is sound and complete for $\mathrm{HA}_{\text {inf }}$.

\section{Conjunctive structural rule is conditionally derivable}

In this section we prove that ConjE (conjunctive structural rule, §6) is derivable from the other rules of $\mathcal{G}_{\text {inf }}$, for all sequents of $\mathrm{L}_{\mathrm{HA}}$ (i.e., $\rightarrow$-free). We will deduce that $\mathcal{G}_{\text {inf }}$ is a conservative extension of $\mathrm{HA}_{\text {inf }}$.

We do not use the particular coding we have chosen for proofs, but only the fact that provability in $\mathcal{G}_{\text {inf }}$ is closed under all rules of Def. 16,17 having finitely many premises. There are also rules of $\mathcal{G}_{\text {inf }}$ with infinitely many premises: the logical rule for $\exists$ in the left-hand-side, and for $\forall$ in the right-hand-side. For these rules, we use a weaker closure property: assume there is a recursive map taking any closed term $t$, and returning a proof of $\Gamma, \mathbf{A}[\mathbf{t}] \vdash D$ in $\mathcal{G}_{\text {inf }}$. Then we conclude $\Gamma, \exists \mathbf{x} . \mathbf{A}[\mathbf{x}] \vdash D$ in $\mathcal{G}_{\text {inf }}$. A dual closure property holds for $\forall$ in the right-hand-side.

Our goal is proving ConjE in $\mathcal{G}_{\text {inf }}$. We introduce an intermediate system $\mathcal{G}_{\mathrm{inf}}{ }^{+}=\left(\mathcal{G}_{\mathrm{inf}}+\mathrm{ConjE}_{\rightarrow}\right)$ between $\mathcal{G}_{\mathrm{inf}}$ and $\mathcal{G}_{\text {inf }}+$ ConjE. Then we start by proving Weakening for $\mathcal{G}_{\text {inf }}, \mathcal{G}_{\text {inf }}{ }^{+}, \mathcal{G}_{\text {inf }}+$ ConjE.

Lemma 5. (Weakening) There is a recursive map turning, for any $A \in$ $\mathrm{L}_{\mathrm{G}}$, any proof $\Pi$ of $\langle\Gamma \vdash D, i\rangle$ in $\mathcal{G}_{\text {inf }}+$ ConjE into a proof $\Pi^{\prime}$ of $\langle\Gamma, A \vdash$ $D, i\rangle$ in $\mathcal{G}_{\text {inf }}$, using the same rules used in $\Pi$.

Proof We define the recursive map by induction over the proof, distinguishing one case for each possible rule at the end of the proof. In each case we apply the inductive hypothesis to the assumptions of the rule, then the rule itself.

The previous Lemma derives Weakening also for $\mathcal{G}_{\text {inf }}, \mathcal{G}_{\text {inf }}{ }^{+}$. We now prove that, whenever we have a proof of $\Gamma \vdash D$ in $\mathcal{G}_{\text {inf }}{ }^{+}$, and a conjunctive occurrence $A(\neq B \rightarrow C)$ in $\Gamma \vdash D$, then we can assume the last rule of the proof is a logical rule with active formula $A$.

Lemma 6. Let $A \in \mathrm{L}_{\mathrm{G} 0}, A^{\prime}<_{1} A$. Then there is some recursive map, taking:

1. any proof of $\langle\Gamma, A \vdash D, i\rangle$ in $\mathcal{G}_{\text {inf }}{ }^{+}$, with $A=\exists x . B, B_{1} \vee B_{2}, a$ (an atom), and returning a proof of $\left\langle\Gamma, A, A^{\prime} \vdash D, i\right\rangle$ in $\mathcal{G}_{\text {inf }}{ }^{+}$.

2. any proof of $\langle\Gamma \vdash A, i\rangle$ in $\mathcal{G}_{\text {inf }}{ }^{+}$, with $A=\neg B$, and returning a proof of $\left\langle\Gamma, A^{\prime} \vdash A, i\right\rangle$ in $\mathcal{G}_{\text {inf }}{ }^{+}$.

3. any proof of $\langle\Gamma \vdash A, i\rangle$ in $\mathcal{G}_{\text {inf }}{ }^{+}$, with $A=\forall x . B, B_{1} \wedge B_{2}, B_{1} \Rightarrow B_{2}$, and returning a proof of $\left\langle\Gamma \vdash A^{\prime}, i\right\rangle$ in $\mathcal{G}_{\text {inf }}{ }^{+}$. 


\section{Proof}

1. By Weakening for $\mathcal{G}_{\text {inf }}{ }^{+}$(Lemma 5).

2. By Weakening for $\mathcal{G}_{\text {inf }}{ }^{+}$(Lemma 5).

3. By induction over the proof. If the last rule has not active occurrence $A$, the thesis is immediate from the induction hypothesis. If the last rule has active formula $A$, then the last rule is logical. Indeed, $A$ is conjunctive and $\neq B_{1} \rightarrow B_{2}$, therefore there is no structural rule in $\mathcal{G}_{\text {inf }}{ }^{+}$inferring $A$. In this case one assumption of the rule is $\left\langle\Gamma \vdash A^{\prime}, i\right\rangle$.

We can now prove that ConjE is a derived rule of $\mathcal{G}_{\text {inf }}{ }^{+}$, that is, that ConjE can be derived from DisjE and ConjE $\rightarrow$.

Lemma 7. (Deriving ConjE in $\mathcal{G}_{\text {inf }}{ }^{+}$) Let $A$ be any formula.

1. There is a recursive map taking any proof of $\langle\Delta \vdash D, i\rangle$ in $\mathcal{G}_{\text {inf }}{ }^{+}$(with active formula $B)$, and returning some proof of $\langle\Delta \vdash D, j\rangle$ in $\mathcal{G}_{\text {inf }}{ }^{+}$ (with active formula $A$ ).

2. $\mathcal{G}_{\text {inf }}+$ ConjE and $\mathcal{G}_{\text {inf }}{ }^{+}$have the same theorems. Besides, there is a recursive map taking any proof of $\langle\Delta \vdash D$, i $\rangle$ in $\mathrm{HA}_{\text {inf }}$, and returning some proof of $\langle\Delta \vdash D, i\rangle$ in $\mathcal{G}_{\text {inf }}{ }^{+}$.

\section{Proof.}

1. By induction over $A$. Assume the thesis holds for any $A^{\prime}<_{1} A$. If $A$ is a disjunctive occurrence, or $A=B \rightarrow C$ is a conjunctive occurrence in the left-hand-side, we apply DisjE or ConjE $\rightarrow$, two rules of $\mathcal{G}_{\text {inf }}{ }^{+}$. Assume $A$ is a conjunctive occurrence and $\neq B \rightarrow C$. There are two cases: $A$ in $\Delta$, and $A=D$.

Assume $\Delta=\Gamma, A$. $A$ is a conjunctive occurrence on the left-handside, therefore $A$ is a disjunctive formula. From $A \neq B \rightarrow C$ we conclude $A=F, \exists x . B, B_{1} \vee B_{2}, a$ (an atom). If $A=F$, then the sequent with active formula $A$ is an axiom. If $A=\exists x . B, B_{1} \vee B_{2}, a$, we apply Lemma 7.1 , and we obtain a family of proofs of $\left\langle\Gamma, A, A^{\prime} \vdash\right.$ $D, i\rangle$ in $\mathcal{G}_{\text {inf }}{ }^{+}$, for any $A^{\prime}<_{1} A$, recursively indexed over $A^{\prime}$. By induction hypothesis over $A^{\prime}$, we can deduce $\Gamma, A, \mathbf{A}^{\prime} \vdash D$ in $\mathcal{G}_{\text {inf }}{ }^{+}$ (with active formula $\mathbf{A}^{\prime}$ ), then $\Gamma, \mathbf{A} \vdash D$ in $\mathcal{G}_{\text {inf }}{ }^{+}$(with active formula A) by a logical rule.

Assume $D=A$. $A$ is a conjunctive occurrence on the right-hand-side, therefore a conjunctive formula, and $A=T, \neg B, \forall x . B, B_{1} \wedge B_{2}, B_{1} \Rightarrow$ $B_{2}$. If $A=T$, the sequent with active formula $A$ is an axiom. If $A=$ $\neg B$, we apply Lemma 7.2 , and we obtain a proof of $\left\langle\Delta, A^{\prime} \vdash A, i\right\rangle$ in $\mathcal{G}_{\text {inf }}{ }^{+}$, for $A^{\prime}=B$, the only $A^{\prime}<_{1} A$. If $A=\forall x . B, B_{1} \wedge B_{2}, B_{1} \Rightarrow B_{2}$, we apply Lemma 7.3 , and we obtain a family of proofs of $\left\langle\Delta \vdash A^{\prime}, i\right\rangle$ in $\mathcal{G}_{\text {inf }}{ }^{+}$, one for each $A^{\prime}<_{1} A$, recursively indexed over $A^{\prime}$. In both subcases, we apply first the induction hypothesis on $A^{\prime}$, in order to deduce $\Delta, \mathbf{A}^{\prime} \vdash A$ or $\Delta \vdash \mathbf{A}^{\prime}$ in $\mathcal{G}_{\text {inf }}{ }^{+}$(with active formula $\mathbf{A}^{\prime}$ ). Then we apply the only logical rule inferring $A$, and we conclude $\Delta \vdash \mathbf{A}$ (with active formula $\mathbf{A}$ ).

2. The rules of $\mathcal{G}_{\text {inf }}+$ ConjE are either the logical rules of $\mathcal{G}_{\text {inf }}{ }^{+}$, or Exchange, and Exchange is derivable in $\mathcal{G}_{\text {inf }}{ }^{+}$by the previous point. We showed that $\mathcal{G}_{\text {inf }}+\mathrm{ConjE}$ and $\mathcal{G}_{\text {inf }}{ }^{+}$have the same theorems. There is only left to show that $\mathcal{G}_{\text {inf }}{ }^{+}$and $\mathcal{G}_{\text {inf }}$ have the same $\rightarrow$-free theorems. 
Definition 19. Denote with $\Delta^{*}$ and $A^{*}$ the sequent and the formula obtained by replacing every negative occurrence $B \rightarrow C$ in the sequent with $B \Rightarrow C$.

Lemma 8. There is a recursive map taking any proof of $\langle\Delta \vdash A, i\rangle$ in $\mathcal{G}_{\text {inf }}{ }^{+}$, and returning a proof of $\left\langle\Delta^{*} \vdash A^{*}, i\right\rangle$ in $\mathcal{G}_{\text {inf }}$.

Proof Sketch. We define the recursive map by induction over the proof, distinguishing one case for each possible rule at the end of the proof. The only non-trivial case is when the last rule of the proof is a proof of $\Gamma, \mathbf{B} \rightarrow \mathbf{C} \vdash A$ in $\mathcal{G}_{\mathrm{inf}}{ }^{+}$, having a negative active occurrence of $\mathbf{B} \rightarrow \mathbf{C}$. We distinguish two subcases, according if the last rule is structural or logical.

- Assume the last rule is a structural rule, necessarily ConjE $\rightarrow$. Remark that $(B \rightarrow C)^{*}=B^{*} \Rightarrow C^{*}$ is a conjunctive formula in the left-hand-side, therefore a disjunctive occurrence. By induction hypothesis we have a proof of $\left\langle\Gamma^{*}, B^{*} \Rightarrow C^{*} \vdash A^{*}, i\right\rangle$ in $\mathcal{G}_{\text {inf }}{ }^{+}$for some $i$. We derive $\Gamma^{*}, \mathbf{B}^{*} \Rightarrow \mathbf{C}^{*} \vdash A^{*}$ in $\mathcal{G}_{\text {inf }}$, with active occurrence $\mathbf{B}^{*} \Rightarrow \mathbf{C}^{*}$, by applying DisjE.

- Assume the last rule is a logical rule. Then we have proofs of $\Gamma, B \rightarrow$ $C \vdash \mathbf{B}$ and $\Gamma, B \rightarrow C, \mathbf{C} \vdash A$ in $\mathcal{G}_{\text {inf }}{ }^{+}$, with active occurrences $\mathbf{B}, \mathbf{C}$. By inductive hypothesis we obtain proofs of $\Gamma^{*}, B^{*} \Rightarrow C^{*} \vdash \mathbf{B}^{*}$ and $\Gamma^{*}, B^{*} \Rightarrow C^{*}, \mathbf{C}^{*} \vdash A^{*}$ in $\mathcal{G}_{\text {inf }}$. We derive first $\Gamma^{*}, B^{*} \Rightarrow C^{*}, B^{*} \rightarrow$ $C^{*} \vdash \mathbf{B}^{*}$ and $\Gamma^{*}, B^{*} \Rightarrow C^{*}, B^{*} \rightarrow C^{*}, \mathbf{C}^{*} \vdash A^{*}$ by Weakening for $\mathcal{G}_{\text {inf }}$. Then we obtain $\Gamma^{*}, B^{*} \Rightarrow C^{*}, \mathbf{B}^{*} \rightarrow \mathbf{C}^{*} \vdash A^{*}$ by the logical rule for $\rightarrow$. Eventually, we obtain $\Gamma^{*}, \mathbf{B}^{*} \Rightarrow \mathbf{C}^{*} \vdash A^{*}$ by the logical rule for $\Rightarrow$.

By combining the last two Lemmas we obtain the theorem:

Theorem 1. (ConjE is derivable from DisjE in $\mathcal{G}_{\text {inf }}$ ). If $\mathcal{G}_{\text {inf }}+$ ConjE proves $\langle\Gamma \vdash A, i\rangle$, and $\Gamma \vdash A$ is in $\mathrm{L}_{\mathrm{HAO}}$, the $\mathcal{G}_{\text {inf }}$ proves $\langle\Gamma \vdash A, i\rangle$.

Proof If $\mathcal{G}_{\text {inf }}+$ ConjE proves $\langle\Gamma \vdash A, i\rangle$, by Lemma 7.2 we deduce that $\mathcal{G}_{\text {inf }}{ }^{+}$proves $\langle\Gamma \vdash A, i\rangle$. By Lemma 8 we deduce that $\mathcal{G}_{\text {inf }}$ proves $\left\langle\Gamma^{*} \vdash\right.$ $\left.A^{*}, i\right\rangle .\langle\Gamma \vdash A, i\rangle$ is $\rightarrow$-free by hypothesis, thus $\left\langle\Gamma^{*} \vdash A^{*}, i\right\rangle=\langle\Gamma \vdash A, i\rangle$.

We end this section with one corollary, $\mathcal{G}_{\text {inf }}$ is a conservative extension of $\mathrm{HA}_{\text {inf }}$, and one easy remark, provability and validity are invariant under index renaming.

Corollary 1. 1. $\mathcal{G}_{\text {inf }}+$ ConjE is a conservative extension of $\mathrm{HA}_{\text {inf }}$

2. $\mathcal{G}_{\text {inf }}$ is a conservative extension of $\mathrm{HA}_{\text {inf }}$.

\section{Proof}

1. $\mathrm{HA}_{\text {inf }}$ is included in $\mathcal{G}_{\mathrm{inf}}+\mathrm{ConjE}$ because the rules for $\Rightarrow$ in $\mathrm{HA}_{\mathrm{inf}}$ are derivable in $\mathcal{G}_{\text {inf }}+$ ConjE, using in this order Weakening for $\mathcal{G}_{\text {inf }}+$ ConjE, then the rules for $\rightarrow$ and for $\Rightarrow$. The reverse inclusion can be proved by taking any proof $\Pi$ in $\mathcal{G}_{\text {inf }}+$ ConjE of a sequent of $\mathrm{L}_{\mathrm{HA}}$, and replacing all $\rightarrow$ by $\Rightarrow$. Then each rule for $\rightarrow$ in $\mathcal{G}_{\text {inf }}+$ ConjE is replaced by the rule for $\Rightarrow$ in $\mathrm{HA}_{\text {inf }}$, each rule for $\Rightarrow$ in $\mathcal{G}_{\text {inf }}+\mathrm{ConjE}$ is replaced by the Contraction rule, which is admissible in $\mathrm{HA}_{\mathrm{inf}}$, and the conclusion of $\Pi$, being in $\mathrm{L}_{\mathrm{HAO}}$, does not change. 
2. By the previous point and Theorem 1 .

Lemma 9. (Index renaming) Assume that $\langle\Gamma \vdash D, i\rangle$ is any sequent of $\mathrm{L}_{\mathrm{G} 0}$, and that $\left\langle\Gamma^{\prime} \vdash D^{\prime}, i^{\prime}\right\rangle$ is its canonical indexing.

1. $\langle\Gamma \vdash D, i\rangle$ is provable in $\mathrm{HA}_{\text {inf }}$ if and only if $\left\langle\Gamma^{\prime} \vdash D^{\prime}, i^{\prime}\right\rangle$ is provable in $\mathrm{HA}_{\text {inf }}$

2. $\mathcal{G} \models\langle\Gamma \vdash D$, i $\rangle$ if and only if $\mathcal{G} \models\left\langle\Gamma^{\prime} \vdash D^{\prime}, i^{\prime}\right\rangle$.

Proof.

1. If two sequents are equal up to indexing of formulas, by index renaming we can turn a proof of the first into a proof of the second.

2. By definition unfolding, we can check that if $\left\langle\Gamma^{\prime} \vdash D^{\prime}, i^{\prime}\right\rangle$ is the canonical indexing of the sequent $\langle\Gamma \vdash D, i\rangle$, then $\operatorname{pos}(\Gamma \vdash D, i)=$ $\operatorname{pos}\left(\Gamma^{\prime} \vdash D^{\prime}, i^{\prime}\right)$. The initial positions of the games $G(\Gamma \vdash D, i), G\left(\Gamma^{\prime} \vdash\right.$ $\left.D^{\prime}, i^{\prime}\right)$ are $\operatorname{pos}(\Gamma \vdash D, i), \operatorname{pos}\left(\Gamma^{\prime} \vdash D^{\prime}, i^{\prime}\right)$, therefore $G(\Gamma \vdash D, i)=$ $G\left(\Gamma^{\prime} \vdash D^{\prime}, i^{\prime}\right)$.

\section{An isomorphism between proofs and strategies}

In this section we prove, as Lorentzen did for his game semantics [6], that our game semantics for $\mathrm{HA}_{\text {inf }}$ is sound and complete. We actually prove more, namely that winning strategies of the semantics and proofs of the variant $\mathcal{G}_{\text {inf }}$ of $\mathrm{HA}_{\text {inf }}$ can be identified. This improvement of Lorentzen's result is inspired by the isomorphism between classical proofs and strategies defined in $([8]){ }^{3}$ A sample of this identification can be seen in $\S 9$. We start proving a relation between positions of a game and the corresponding sequents.

Lemma 10. Let $P$ be any position. Let $s_{1} A_{1}, \ldots, s_{n} A_{n}$ be the list of judgements in $P$. Assume $\langle\Gamma \vdash D, i\rangle=\operatorname{seq}(P)$. Then:

1. ( $j$ index of $\operatorname{seq}(P)) \Leftrightarrow(j$ backtracking position of $P)$.

2. $P, \operatorname{seq}(P)$ have the same maximum index.

3. $\Gamma=\left\{\left\langle k, A_{k}\right\rangle \mid s_{k}=\mathrm{f}\right.$. $\}$ ( = all negative judgements, with the index they have in $P)$.

4. $D=\left\{\left\langle k, A_{k}\right\rangle\right\}$, for the last $k$ such that $s_{k}=\mathrm{t}$. (= the last positive judgement, with its index in $P)$.

5. $i=$ the index of the active move of $P$.

Proof. We will prove point $3,4,5$ by simultaneous induction over the length of $P$. Points 1, 2 are implied by points 3,4 . Indeed, the set of bckindexes of $P$ consists of all indexes of negative judgements, and of the last positive judgement, and by 3,4 , of all indexes of $\operatorname{seq}(P) . P, \operatorname{seq}(P)$ have the same maximum index, because the largest index of $P$ is equal to the largest bck-index of $P$.

If $P=$ t. $A$, then $3,4,5$ are immediate from the definition of seq. Assume $P=Q, m$, for some position $Q$ with $n>0$ judgements, and some $m \in$

\footnotetext{
${ }^{3}$ Another proof-strategy isomorphism result of the same kind can be found in [3].
} 
Moves. Let $n=$ the maximum index of $\operatorname{seq}(Q)=($ by ind.hyp. on $Q)$ the maximum index of $Q$.

Assume $m=s A$. Then $s A$ has index $n+1$ in $P, \operatorname{seq}(P)$. If $s=\mathrm{f}$., then $s A$ is added to the left-hand-side of $\operatorname{seq}(Q)$, and is added to the negative judgements of $Q$. If $s=\mathrm{t}$., then $s A$ is replaced to the right-hand-side of seq $(Q)$, and is replaced to the last positive judgement of $Q$. We deduce $3,4 . n+1$ is the active occurrence of seq $(P)$ and the active move of $P$. We deduce 5 .

Assume $m=\operatorname{bck}(j)$. Then $\operatorname{seq}(P), \operatorname{seq}(Q)$ have the same left- and righthand side, and $P, Q$ have the same sub-list of judgements. Since $Q$ satisfies 3,4 , then $P$ satisfies 3,4 . The active occurrence of $\operatorname{seq}(P)$ is $j$ if and only if $j$ is an index of $\operatorname{seq}(Q)$, it is $n$ o.w.. The active move of $P$ is $j$ if and only if $j$ is a bck-index of $Q$, it is $n$ o.w. By ind. hyp. on $Q$, we deduce that the active occurrence of $\operatorname{seq}(P)$ and the active move of $P$ are the same. We conclude 5 .

Isomorphism Theorem says that recursive winning strategies for $\mathcal{E}$ and proofs of $\mathcal{G}_{\text {inf }}$ are isomorphic, and in fact, with the coding we have chosen, identical.

Theorem 2. (Isomorphism theorem) Fix any pointed sequent $S=\langle\Gamma \vdash$ $A, i\rangle$ having a canonical indexing and $n>0$ formulas. Then:

( $\Pi$ is a proof of $\langle\Gamma \vdash A, i\rangle$ in $\left.\mathcal{G}_{\text {inf }}\right) \Leftrightarrow(\Pi$ is a recursive winning strategy for $\mathcal{E}$ on $G(\Gamma \vdash A, i)$ )

Proof. Let $P=\operatorname{pos}(\Gamma \vdash D, i)$. By Lemma 1 , and the fact that $S=\langle\Gamma \vdash$ $D, i\rangle$ has a canonical indexing, we have $\operatorname{seq}(P)=S$. By Lemma $4, \Pi$ is a proof of $S$ in $\mathcal{G}_{\text {inf }}$ if and only if $\Pi$ is a recursive well-founded tree, and, for all $x \in \Pi$, if $\operatorname{seq}_{S}(x)$ has active formula $A$ of sign $s$, then

1. either $s A$ is disjunctive and Children $(x)=\{\operatorname{bck}(j)\}$ for some $j$ index of $\operatorname{seq}_{S}(x)$;

2. or $s A$ is disjunctive and Children $(x)=\left\{s^{\prime} A^{\prime}\right\}$, for some $s^{\prime} A^{\prime}<_{1} s A$;

3. or $s A$ is conjunctive and Children $(x)=\left\{s^{\prime} A^{\prime} \mid s^{\prime} A^{\prime}<_{1} s A\right\}$.

By definition, $\operatorname{seq}_{S}(x)=S+x=\operatorname{seq}(P)+x=\operatorname{seq}(P @ x)$. Therefore the predicate " $j$ index of $\operatorname{seq}_{S}(x)$ " in the first clause is equivalent to " $j$ index of seq $(P @ x)$ ". By Lemma 10.1 applied to $(j$ index of seq $(P @ x))$, clause 1 above is equivalent to:

1. Children $(x)=\{\operatorname{bck}(j)\}$ for some $j$ bck-index of $P @ x$.

By Lemma 10.5, the hypothesis " $A$ active formula of seq $(P @ x)$, of sign $s$ " is equivalent to: " $s A$ active move of $P @ x$ ". By Lemma 2, the assumption about $\Pi$, if reformulated in this way, is equivalent to the definition of recursive winning strategy for $\mathcal{E}$ on $G_{P}=G(\Gamma \vdash D, i)$.

Soundness and Completeness are immediate from the Isomorphism Theorem.

Theorem 3. (Completeness Theorem) Let $\langle\Gamma \vdash A, i\rangle$ be any pointed sequent of $\mathrm{L}_{\mathrm{HaO}}$. Then:

(HA $_{\text {inf }}$ proves $\left.\langle\Gamma \vdash A, i\rangle\right) \Leftrightarrow \mathcal{G} \models\langle\Gamma \vdash A, i\rangle$. 
Proof. In view of Lemma 9, we can assume that $\langle\Gamma \vdash D, i\rangle$ has a canonical indexing, so we can apply Theorem 2. Now suppose $\langle\Gamma \vdash A, i\rangle$ has a proof $\Pi$ in $\mathrm{HA}_{\text {inf }}$. By Corollary $1,\langle\Gamma \vdash A, i\rangle$ has a proof $\Pi^{\prime}$ in $\mathcal{G}_{\text {inf }}$. By Theorem 2, $\Pi^{\prime}$ is also a recursive winning strategy for $\mathcal{E}$ on $G(\Gamma \vdash A, i)$. Suppose the converse: $\mathcal{E}$ has some recursive winning strategy $\sigma$ on $G(\Gamma \vdash A, i)$. By Theorem 2 again, $\sigma$ is a proof of $\langle\Gamma \vdash A, i\rangle$ in $\mathcal{G}_{\text {inf }}$, and by Corollary 1 we have a proof in in $\mathrm{HA}_{\text {inf }}$.

As a consequence of the main Theorem, we check that our game semantics does not validate some true positive formula, while it validates the negation of all false positive formulas.

Corollary 2. Let $A \in \mathrm{L}_{0}^{+}$(i.e., $A$ is $\neg, \Rightarrow, \rightarrow$-free).

1. For some true $A$ we have $\neg(\mathcal{G} \models A)$.

2. $\mathcal{G} \models A \vdash F$ if and only if $A$ is false (in the standard model of $N$ ).

\section{Proof}

1. Let $\forall y . P(x, y)$ be any non-decidable predicate, and $P^{\perp}$ be the complement of $P$. Then $A=\forall x \cdot\left(\forall y \cdot P(x, y) \vee \exists y \cdot P^{\perp}(x, y)\right)$ is an instance of Excluded Middle. $A$ is true, but it cannot be proved in $\mathrm{HA}_{\mathrm{inf}}$. By Theorem 3 we conclude $\neg(\mathcal{G} \models A)$.

2. $A \vdash F$ is a theorem of $\mathrm{HA}_{\mathrm{inf}}$ if and only if it is a theorem of $\mathrm{PA}_{\mathrm{inf}}$, classical arithmetic with $\omega$-rule, because the rules of $\mathrm{HA}_{\text {inf }}, \mathrm{PA}_{\text {inf }}$ for positive formulas in the left-hand-side are the same. By completeness of $\mathrm{HA}_{\mathrm{inf}}$ w.r.t. game semantics (Theorem 3), and of $\mathrm{PA}_{\mathrm{inf}}$ w.r.t. first order semantics (folklore), we conclude $\mathcal{G} \models A \vdash F$ if and only if $A \vdash F$ is true, that is, if and only if $A$ is false.

\section{References}

[1] S. Berardi. Semantics for Intuitionistic Arithmetic based on Tarski Games with retractable moves, Technical Report, Turin University, January 2007. http://www.di.unito.it/ stefano/BerardiGamesForIntuitionisticLogic-200\%-01.pdf.

[2] S. Berardi, Th. Coquand., S. Hayashi. Games with 1-backtracking, Proceedings of GaLop, Edinburgh, April 2005.

[3] S. Berardi, Y. Yamagata. A sequent calculus for 1-backtracking, Technical Report, Turin University, December 2005. CL\&C 2006. Submitted to the special issue of APAL for the congress. http://www.di.unito.it/ stefano/Yamagata-Berardi-report.pdf.

[4] Th. Coquand. A semantics of evidence for classical arithmetic (preliminary version), Proceedings of the Second Workshop on Logical Frameworks, Edinburgh, 1991, Gérard Huet, Gordon Plotkin and Claire Jones, eds.. http://www.dcs.ed.ac.uk/home/lego/html/papers.html

[5] Th. Coquand. A semantics of evidence for classical arithmetic. Journal of Symbolic Logic 60 (1995), pp. 325-337.

[6] W. Felscher. Lorentzen's game semantics Handbook of Philosophical Logic, 2nd Edition, Volume 5, pages 115-145 2002 Kluwer Academic Publisher, the Netherlands. 
[7] Hayashi, S.: Can proofs be animated by games?, in P. Urzyczyn ed., TLCA 2005, LNCS 3461, pp.11-22, 2005, invited paper.

[8] H. Herbelin, Sequents qu'on calcule: de l'interpretation du calcul des sequents comme calcul de lambda-termes et comme calcul de strategies gagnantes, Ph. D. thesis, University of Paris VII, 1995.

[9] W. Hodges, "Logic and Games", The Stanford Encyclopedia of Philosophy (Winter 2004), Edward N. Zalta (ed.).

http://plato.stanford.edu/archives/win2004/entries/logic-games/

[10] M. Hyland and L. Ong", On full abstraction for PCF,Information and Computation, 2000, volume 163, pages 285-408.

\section{A proof of $\exists x \cdot P(x) \wedge \exists y \cdot Q(y) \Rightarrow$ $\exists x . \exists y \cdot(P(x) \wedge Q(y))$}

Let $A=\exists x \cdot P(x) \wedge \exists y \cdot Q(y)$ and $B=\exists x \cdot \exists y .(P(x) \wedge Q(y))$. In this section we include a recursive winning strategy $\sigma$ for t. $A \Rightarrow B$. By Theorem 2, $\sigma$ is also a proof of $A \Rightarrow B$ in $\mathrm{HA}_{\text {inf }}$. In $\S 10$ we include a play following this strategy $\sigma$.

In the proof-tree below, we include the sequent labelling each node of the tree, and print boldface the active occurrence of each sequent. We include the move associated to each child of each node, and we add a label " 1 :", "2 :", . . in front of the logical move number $1,2, \ldots$ of the strategy. The strategy-tree we draw is incomplete: we should add one branch for each value of $t$ and each value of $u$. In the particular branches included in the picture, we assumed that $P(t)$ and $Q(u)$ are true. Branches with $P(t)$ or $Q(u)$ false (not included below) are slightly different. We leave the reader check that this strategy-tree is also a proof-tree of $\mathrm{HA}_{\text {inf }}$, without the rule ConjE. Indeed, each node is the conclusion of some rule of $\mathrm{HA}_{\mathrm{inf}}$.

$$
\begin{aligned}
& \overline{A, \exists x \cdot P(x), P(t), T, \exists y \cdot Q(y), Q(u), T \vdash \mathbf{T}} \quad \overline{A, \exists x \cdot P(x), P(t), T, \exists y \cdot Q(y), Q(u), T \vdash \mathbf{T}} \\
& \overline{A, \exists x . P(x), P(t), T, \exists y \cdot Q(y), Q(u), T \vdash \mathbf{P}(\mathbf{t})} \overline{A, \exists x . P(x), P(t), T, \exists y \cdot Q(y), Q(u), T \vdash \mathbf{Q}(\mathbf{u})} 14: \text { t.T } 13: \text { t.P(t) or t. }
\end{aligned}
$$

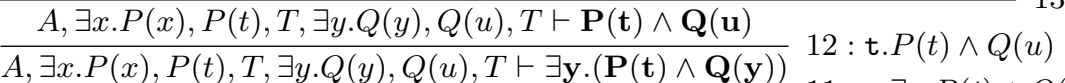

$$
\begin{aligned}
& A, \exists x . P(x), P(t), T, \exists y \cdot Q(y), Q(u), T \vdash \mathbf{B} 11: \text { t. } \exists y \cdot P(t) \wedge Q(y) \\
& \overline{A, \exists x . P(x), P(t), T, \exists y \cdot Q(y), Q(u), T \vdash \mathbf{A} \rightarrow \mathbf{B}} 10: \text { t.B } \\
& \overline{A, \exists x \cdot P(x), P(t), T, \exists y \cdot Q(y), Q(u), \mathbf{T} \vdash A \rightarrow B} \operatorname{bck}(2)
\end{aligned}
$$

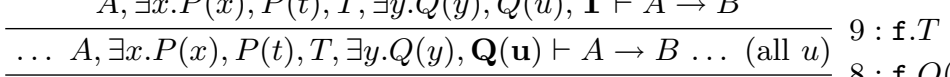

$$
\begin{aligned}
& \frac{A, \exists x . P(x), P(t), T, \exists \mathbf{y} \cdot \mathbf{Q}(\mathbf{y}) \vdash A \rightarrow B}{\mathbf{A}, \exists x \cdot P(x), P(t), T \vdash A \rightarrow B} 7: \mathbf{f} . \exists y \cdot Q(y) \\
& \frac{\mathbf{A}, \exists x . P(x), P(t), T \vdash A \rightarrow B}{A, \exists x \cdot P(x), P(t), T \vdash A \rightarrow B} \operatorname{bck}(3) \\
& \begin{array}{cl}
\frac{A, \exists x . P(x), P(t), \mathbf{T} \vdash A \rightarrow B}{\ldots A, \exists x . P(x), \mathbf{P}(\mathbf{t}) \vdash A \rightarrow B \ldots(\text { all } t)} & 6: \mathrm{f} . T \\
& 5: \mathrm{f} . P(t)
\end{array} \\
& \frac{A, \exists \mathbf{x} . \mathbf{P}(\mathbf{x}) \vdash A \rightarrow B}{\mathbf{A} \vdash A \rightarrow B} 4: \mathbf{f} . \exists x . P(x) \\
& \frac{\mathbf{A} \vdash A \rightarrow B}{+A \rightarrow \mathbf{B}} 3: \mathrm{f} . A \\
& \stackrel{\vdash \mathbf{A} \rightarrow \mathbf{B}}{\vdash \mathbf{A} \rightarrow \mathbf{B}} \quad 2: \mathrm{t} . A \rightarrow B
\end{aligned}
$$




\section{An example of game interpretation}

In this section we include one example of play following the strategy $\sigma$ of $\S 9$. More examples of plays can be found in the section about game semantics in the web page of the author(look for the subsection about intuitionistic implication).

The game is t. $A \Rightarrow B$, with

$$
A=\exists x \cdot P(x) \wedge \exists y \cdot Q(y) \quad \text { and } \quad B=\exists x \cdot \exists y \cdot(P(x) \wedge Q(y))
$$

Here is (a simplified picture of) the sub-judgement tree of t. $A \Rightarrow B$. The tree in the picture is incomplete: we should add one branch for each closed term $t, u$ of the language, and some $T$ or $F$ below each atom, according if the atom is true or false.

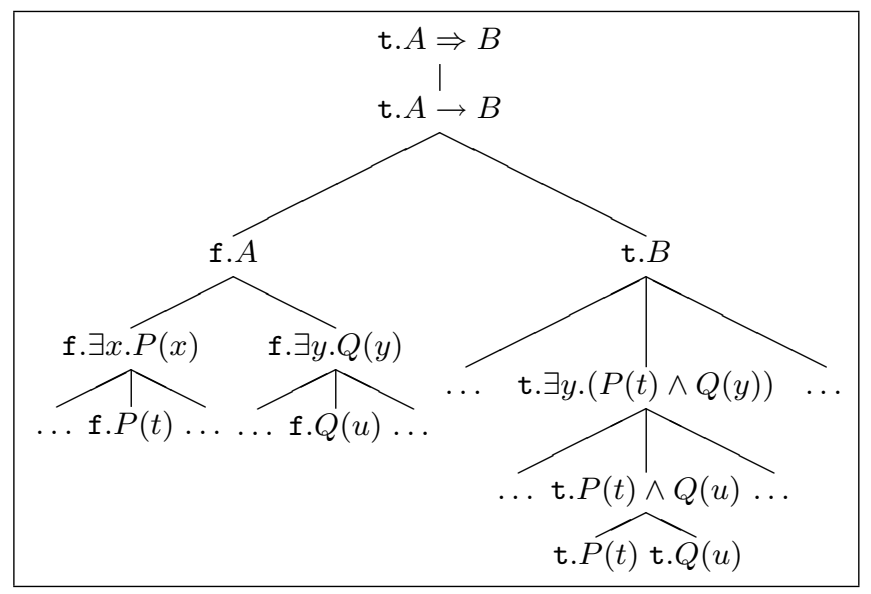

We informally outline the behavior of the recursive winning strategy $\sigma$ for $\mathcal{E}$ on t. $A \Rightarrow B$. $\mathcal{E}$ first asks $\mathcal{A}$ for some $x$ such that $P(x)$, then for some $y$ such that $Q(y)$. In the case $\mathcal{A}$ cannot provide $x$ as required, or cannot provide $y$ as required, $\mathcal{E}$ wins. If $\mathcal{A}$ can provide both $x$ and $y$, $\mathcal{E}$ returns the pair $x, y$ and wins, because we have $P(x) \wedge Q(y)$. In this section we include a generic play in which $\mathcal{E}$ follows $\sigma$. At each step, we draw all previous positions of the play in tree form, numbered $1,2,3, \ldots$. In our picture, each branch of the tree is state of some thread in a given step of the play. The first child of a given node is an ordinary move of Tarski play. Each time $\mathcal{E}$ backtracks to the node, she adds one more child to it, and the thread forks (the first example is logical move 7 below). We dot all positions $\mathcal{E}$ can backtrack to: all negative judgements by $\ominus$, the last positive judgement by $\oplus$. We make bigger the dot corresponding to the active move: $\ominus, \oplus$. There is one Abelard guarding each node of the tree. Whenever the play moves from a node to one of its children, one Abelard is detached guarding the previous node, and waiting, possibly forever, for $\mathcal{E}$ to come back to this node. At each step, $\mathcal{E}$ is facing the Abelard guarding the node with the big dot. 
We assume that $P(t), Q(u)$ are true for the particular $t, u$ we include in the picture. For each step of the play we include the associated sequent $\Gamma \vdash D$, with the active occurrence boldface: say, $\Gamma \vdash \mathbf{D}$, if $D$ is the active occurrence. $\Gamma$ consists of all formulas dotted $\ominus$, and $D$ is the only formula dotted $\oplus$. The active occurrence of $\Gamma \vdash D$ is the only formula having a big dot: $\ominus, \oplus$. Now we draw a generic play in which $\mathcal{E}$ follows $\sigma$.

- Logical move 1, or initial position: t. $A \Rightarrow B$, an intuitionistic implication.

$$
\text { t. } A \Rightarrow B
$$

Associated sequent: $\vdash \mathbf{A} \Rightarrow \mathbf{B}$

- Logical move 2 , by $\mathcal{E}:$ t. $A \rightarrow B$, the only subformula of $A \Rightarrow B$.

$$
\begin{aligned}
& \text { 七. } A \underset{1}{\Rightarrow} B \\
& \text { 七. } A \underset{\text { । }}{\rightarrow} B
\end{aligned}
$$

Associated sequent: $\vdash \mathbf{A} \rightarrow \mathbf{B}$

Position 1 (t. $A \Rightarrow B$ ) is no more dotted, it is no more the last positive judgement. $\mathcal{E}$ cannot anymore come back to it.

- Logical move 3 , by $\mathcal{E}:$ f.A. $\mathcal{E}$ asks $\mathcal{A}$ to defend the truth of $A$.

$$
\begin{gathered}
\text { t. } A \underset{1}{\Rightarrow} B \\
\text { t. } A \underset{2}{\operatorname{l}} \underset{\text { । }}{\rightarrow} B \\
\text { f. } A \\
\Theta \oplus
\end{gathered}
$$

Associated sequent: $\mathbf{A} \vdash A \rightarrow B$

- Logical move 4 , by $\mathcal{E}$ : f. $\exists x . P(x) . \mathcal{E}$ asks $\mathcal{A}$ to provide some $x$ such that $P(x)$.

$$
\begin{array}{|c|}
\hline \text { t. } A \Rightarrow B \\
\text { t. } A \underset{\text { ! }}{\rightarrow} B \\
\text { । } \\
\text { f.A } \\
3 \ominus \\
\text { । } \\
\text { f. } \exists . P(x) \\
4 \ominus \\
\hline
\end{array}
$$

Associated sequent: $A, \exists \mathbf{x} . \mathbf{P}(\mathbf{x}) \vdash A \rightarrow B$

- Logical move 5 , by $\mathcal{A}$ : any f.P $(t)$. $\mathcal{A}$ provides some $x=t$. 


$$
\begin{aligned}
& \text { t. } A \underset{1}{\Rightarrow} B \\
& \underset{2 \oplus}{\mathrm{t} .} \stackrel{\mathrm{l}}{\rightarrow} B \\
& \text { f.A } \\
& 3 \ominus \\
& \text { f. } \exists x . P(x) \\
& 4 \ominus \\
& \stackrel{1}{f . P(t)} \\
& 5 \ominus
\end{aligned}
$$

Associated sequent: $A, \exists x . P(x), \mathbf{P}(\mathbf{t}) \vdash A \rightarrow B$

- Logical move 6, by $\mathcal{A}$ : $\mathrm{f} . F$ if $P(t)$ is false, and f.T if $P(t)$ is true. In the first case, in the next step $\mathcal{A}$ should move from f.F, he cannot and he loses. From now on, we assume that $P(t)$ is true.

$$
\begin{array}{|c|}
\hline \text { t. } A \Rightarrow B \\
1 \\
\text { t. } A \underset{ }{2} \rightarrow \\
\text { । } \\
\text { f. } A \\
3 \ominus \\
\text { । } \\
\text { f. } x . P(x) \\
4 \ominus \\
\text { । } \\
\text { f.P }(t) \\
5 \ominus \\
\text { । } \\
6 . T \\
6 \ominus
\end{array}
$$

Associated sequent: $A, \exists x . P(x), P(t), \mathbf{T} \vdash A \rightarrow B$. In the next step $\mathcal{E}$ should move from $\mathrm{f} . T$, she cannot and she is forced to backtrack.

- Backtrack to f.A, the negative judgement of index 3 , by $\mathcal{E}$ : bck(3)

$$
\begin{aligned}
& \underset{1 \oplus}{\text { t. } A \Rightarrow B} \\
& \text { t. } \underset{2 \oplus}{\stackrel{\text { I }}{\rightarrow}} B \\
& \text { f. } A \\
& 3 \stackrel{\text { f. } A}{\ominus} \\
& \stackrel{\text { f }}{ } \exists x . P(x) \\
& 4 \ominus \\
& \stackrel{\stackrel{1}{f .} P(t)}{ } \\
& 5 \ominus \\
& \text { f.T }
\end{aligned}
$$


Only the position of the big dot changed. Now the big dot is over the logical move $3 . \mathcal{E}$ is facing the Abelard guarding the node 3. Associated sequent: A, $\exists x . P(x), P(t), T \vdash A \rightarrow B$

- Logical move 7 , by $\mathcal{E}$ : f. $\exists y \cdot Q(y) . \mathcal{E}$ asks $\mathcal{A}$ to provide some $y$ such that $Q(y)$. The thread from t. $A \Rightarrow B$ forks, and a new thread starts from $\mathbf{f} . \exists y \cdot Q(y)$.

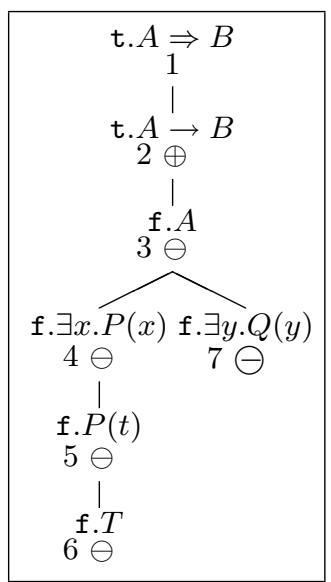

Associated sequent: $A, \exists x \cdot P(x), P(t), T, \exists \mathbf{y} \cdot \mathbf{Q}(\mathbf{y}) \vdash A \rightarrow B$

- Logical move 8, by $\mathcal{A}$ : any f.Q(u).

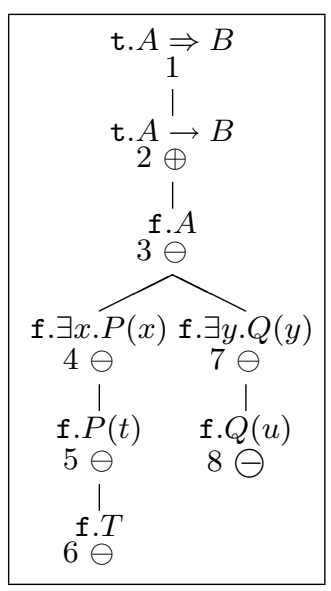

Associated sequent: $A, \exists x . P(x), P(t), T, \exists y \cdot Q(y), \mathbf{Q}(\mathbf{u}) \vdash A \rightarrow B$

- Logical move 9, by $\mathcal{A}$ : f.F if $Q(u)$ is false, and f.T if $Q(u)$ is true. In the first case, in the next step $\mathcal{A}$ should move from f.F, he cannot and he loses. From now on, we assume that $Q(u)$ is true. 


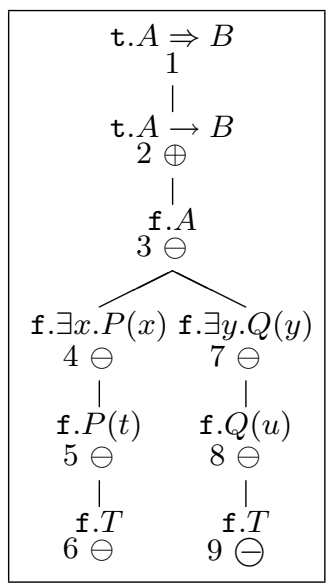

Associated sequent: $A, \exists x . P(x), P(t), T, \exists y \cdot Q(y), Q(u), \mathbf{T} \vdash A \rightarrow B$. In the next step $\mathcal{E}$ should move from f.T, she cannot and she is forced to backtrack.

- Backtrack by $\mathcal{E}$, to t. $A \rightarrow B$, the last positive judgement, of index 2: $\operatorname{bck}(2)$

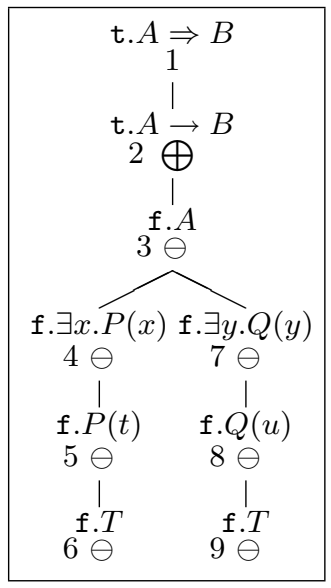

Only the position of the big dot changed. Now the big dot is over the logical move 2. $\mathcal{E}$ is facing the Abelard guarding the node 2. Associated sequent: $A, \exists x . P(x), P(t), T, \exists y \cdot Q(y), Q(u), T \vdash \mathbf{A} \rightarrow \mathbf{B}$

- Logical move 10, by $\mathcal{E}$ : t.B. After attacking $A$ twice, $\mathcal{E}$ claims she has enough information to defend $B$. The thread from t. $A \Rightarrow B$ forks again, and a new thread starts from t.B. This latter cannot fork, because $B$ is a positive formula (see $\S 4.3$ ). 


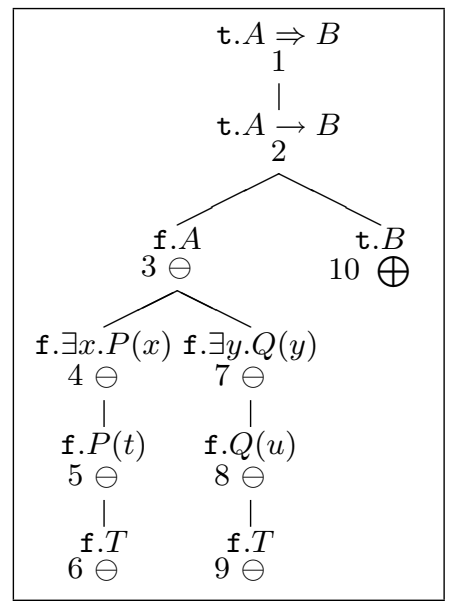

Positions 1,2 are no more dotted, they are no more the last positive judgement. Associated sequent: $A, \exists x . P(x), P(t), T, \exists y \cdot Q(y), Q(u), T \vdash$ B

- Logical move 11, by $\mathcal{E}:$ t. $\exists y .(P(t) \wedge Q(y))$. $\mathcal{E}$ chooses $x=t$.

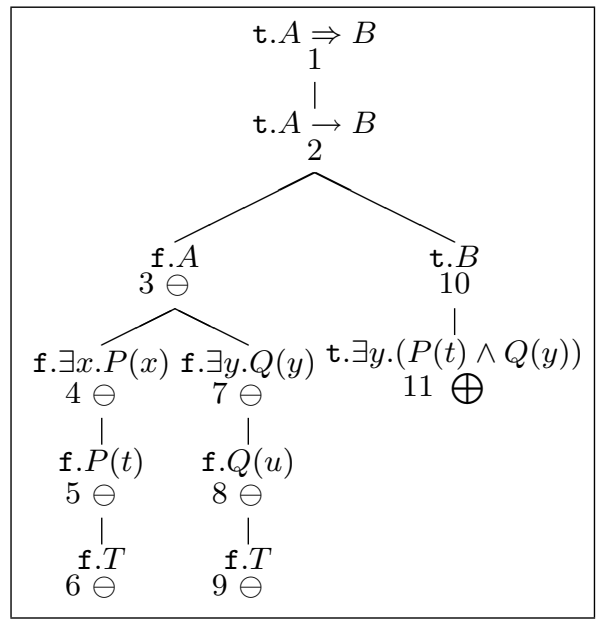

Associated sequent: $A, \exists x . P(x), P(t), T, \exists y \cdot Q(y), Q(u), T \vdash \exists \mathbf{y} \cdot(\mathbf{P}(\mathbf{t}) \wedge \mathbf{Q}(\mathbf{y}))$

Position 1,2, 10 are no more dotted, for the usual reason. Therefore $\mathcal{E}$ cannot backtrack to t. $B$. Her choice $x=t$ must be the best possible on the first try.

- Logical move 12 , by $\mathcal{E}$ : t. $P(t) \wedge Q(u)$. $\mathcal{E}$ chooses $y=u$. 


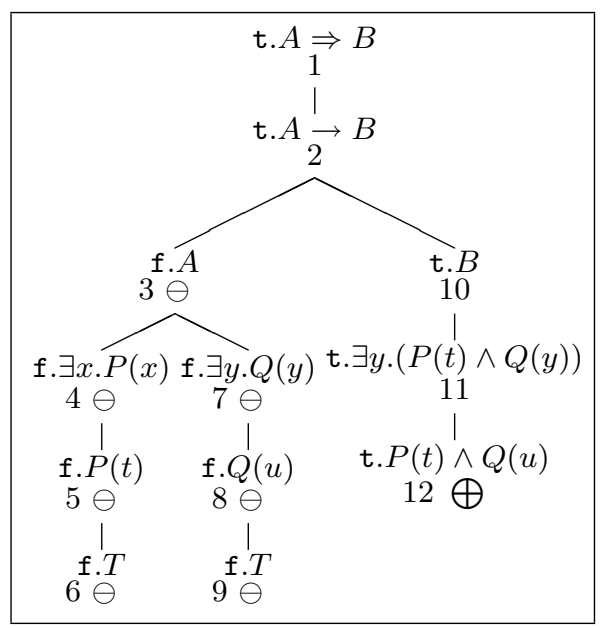

Associated sequent: $A, \exists x . P(x), P(t), T, \exists y . Q(y), Q(u), T \vdash \mathbf{P}(\mathbf{t}) \wedge \mathbf{Q}(\mathbf{u})$

Position 1,2,10, 11 are no more dotted, for the usual reason. Therefore $\mathcal{E}$ cannot backtrack to t. $\exists y .(P(t) \wedge Q(y))$. Her choice $y=u$ must be the best possible on the first try.

- Logical move 13 , by $\mathcal{A}$ : either t.P(t), or t. $Q(t)$. $\mathcal{A}$ checks whether the pair $x=t, y=u$ satisfies $P(x) \wedge Q(y)$, by checking either $P(x)$, or $Q(y)$. Assume the Abelard facing $\mathcal{E}$ moves t. $P(t)$.

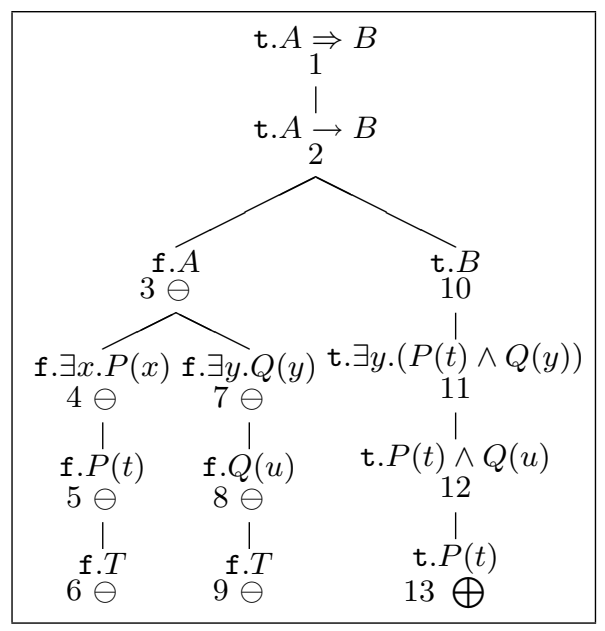

Associated sequent: $A, \exists x . P(x), P(t), T, \exists y \cdot Q(y), Q(u), T \vdash \mathbf{P}(\mathbf{t})$

All proper ancestors of position 13 are no more dotted. Therefore the rightmost thread cannot fork.

- Logical move 14 , by $\mathcal{E}$ : t.T (we assumed that $P(t)$ is true). 


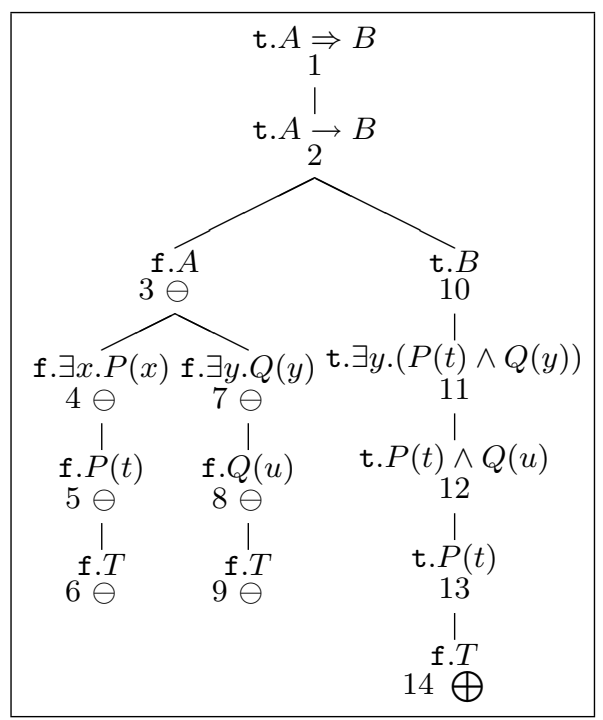

Associated sequent: $A, \exists x . P(x), P(t), T, \exists y \cdot Q(y), Q(u), T \vdash \mathbf{T}$.

All proper ancestors of position 14 are no more dotted.

- In the next step, $\mathcal{A}$ should move from t.T, but he cannot. $\mathcal{A}$ gives up: drop 